\title{
Measurements and simulations of polarization states of underwater light in clear oceanic waters
}

\author{
Yu You, ${ }^{1}$ Alberto Tonizzo, ${ }^{2}$ Alexander A. Gilerson, ${ }^{2}$ Molly E. Cummings, ${ }^{3}$ \\ Parrish Brady, ${ }^{3}$ James M. Sullivan, ${ }^{4,5}$ Michael S. Twardowski, ${ }^{4}$ \\ Heidi M. Dierssen, ${ }^{6}$ Samir A. Ahmed, ${ }^{2}$ \\ and George W. Kattawar ${ }^{1, *}$ \\ 'Department of Physics and Astronomy, Texas A\&M University, College Station, Texas 77843-4242, USA \\ ${ }^{2}$ Optical Remote Sensing Laboratory, The City College of the City University of New York, \\ New York, New York 10031, USA \\ ${ }^{3}$ Section of Integrative Biology, University of Texas, Austin, Texas 78712, USA \\ ${ }^{4}$ WET Labs Inc., 70 Dean Knauss Road, Narragansett, Rhode Island 02882-1197, USA \\ ${ }^{5}$ University of Rhode Island, Graduate School of Oceanography, South Ferry Road, \\ Narragansett, Rhode Island 02882-1197, USA \\ ${ }^{6}$ Department of Marine Sciences, University of Connecticut Avery Point, 1080 Shennecossett Road, \\ Groton, Connecticut 06340-6048, USA \\ *Corresponding author: kattawar@tamu.edu
}

Received 17 December 2010; revised 22 May 2011; accepted 22 July 2011; posted 22 July 2011 (Doc. ID 139780); published 19 August 2011

\begin{abstract}
Polarization states of the underwater light field were measured by a hyperspectral and multiangular polarimeter and a video polarimeter under various atmospheric, surface, and water conditions, as well as solar and viewing geometries, in clear oceanic waters near Port Aransas, Texas. Some of the first comprehensive comparisons were made between the measured polarized light, including the degree and angle of linear polarization and linear Stokes parameters $(Q$ and $U)$, and those from Monte Carlo simulations that used concurrently measured water inherent optical properties and particle volume scattering functions as input. For selected wavelengths in the visible spectrum, measured and modelsimulated polarization characteristics were found to be consistent in most cases. Measured degree and angle of linear polarization are found to be largely determined by an in-water single-scattering model. Model simulations suggest that the degree of linear polarization (DoLP) at horizontal viewing directions is highly dependent on the viewing azimuth angle for a low solar elevation. This implies that animals can use the DoLP signal for orientation. (C) 2011 Optical Society of America
\end{abstract}

OCIS codes: $\quad 010.4458,010.5620,290.5855$.

\section{Introduction}

For over 50 years, the polarized nature of the underwater light field has been the subject of extensive research, as it has been found to be detected and utilized by a variety of marine animals. Some of the first

0003-6935/11/244873-21\$15.00/0

(C) 2011 Optical Society of America measurements of the polarized underwater light field were reported by Waterman [1], Waterman and Westell [2], and Ivanoff and Waterman [3], who pointed out that underwater polarization patterns are important for marine animals and discussed the dependence of the polarization states on the solar position and viewing directions. Some other early measurements of the underwater polarization include Ivanoff et al.'s [4] measurement of the highly 
polarized light field (75\%-80\%) in clear waters near Bermuda, and laboratory measurements by Timofeeva [5] that showed a degree of (linear) polarization (DoL̄P) of up to $40 \%$ in turbid waters. Also, as shown by Chami [6] , underwater polarization can be used to study the optical signature of inorganic particles in coastal waters from remote sensing.

In an effort to understand the impact of polarization states on marine animals, measurement of multispectral underwater polarization has again become popular in the experimental biology community in recent years [7-13]. These studies reported measurements of not only the DoLP, but also the orientation of the electric field vector (e-vector), i.e., the angle of (linear) polarization (AoLP), as evidence showed that some marine animals are sensitive to both quantities. The dependence of both the DoLP and the AoLP on solar positions, viewing angles, and wavelengths were discussed in these studies. The importance of information about water optical properties together with the knowledge of the distributions of DoLP and e-vector was especially emphasized for the efficient evaluation of possible mechanisms of animal vision and communications, as well as for the understanding and development of polarization techniques that improve underwater visibility and orientation. Most recently, a hyperspectral and multiangular analysis of polarized light in coastal waters was reported by Tonizzo et al. [14], who discussed the impact of atmospheric conditions and water compositions on underwater DoLP.

Simulations of the underwater polarized light field were conducted when numerical models that solve the vector radiative transfer equation became available in the 1970s. Such models include the Monte Carlo [15-17] and multicomponent approximation methods [18]. Given appropriate input, including solar irradiance, atmospheric conditions, aerosol loading, and water inherent optical properties (IOPs), model simulations can reproduce both radiance and polarization states from field measurements. Comparisons between simulated and measured underwater polarized light fields have been reported by, for example, Adams et al. [19] for one wavelength, and Tonizzo et al. [14] for the whole visible spectrum, and consistencies have been observed in the DoLP in both studies. However, as far as the authors are aware, no comparisons have been published for the Stokes parameters or the e-vector orientation (AoLP).

There were also no comprehensive comparisons among measured and simulated DoLP for various illuminations, viewing conditions, water parameters, sea states, or depths, which really are necessary for further reliable applications of the Monte Carlo simulations in the broad analysis of underwater polarization fields. Such comparisons require simultaneous high quality measurements of the Stokes vector components, IOPs, and volume scattering functions; the instrumentation for such combination of measurements has just recently emerged.
In this paper, we present recent measurements of the underwater polarized light field from clear ocean waters off Port Aransas, Texas, using two instruments: a hyperspectral and multiangular polarimeter and a video polarimeter. The latter is much easier to operate, provides spatial information rather than point sampling, and has never before been included in underwater polarization models, to the best of our knowledge. In addition to the light field measurements, water IOPs, including particulate volume scattering function (VSF) measurements were conducted. We then compare the measured polarization states with their counterparts from numerical simulations using a Monte Carlo radiative transfer model, where the measured IOPs and VSFs were used as input. Unlike previous studies that were limited to comparisons of measured and simulated DoLP, here we aim to provide a full description of the linear polarization state, including the DoLP and AoLP, as well as the linear polarization part of the Stokes vector, i.e., the $Q$ and $U$ components, for several wavelengths in the visible spectrum. Here, comparisons of measured and model-simulated polarized light fields show general consistencies between the two. Additional sensitivity analyses investigate the DoLP and AoLP patterns with in-water single-scattering models, as well as the polarization dependence due to various input parameters, including the solar elevation, depth, and water turbidity.

The paper is organized as follows. In Section 2, a brief theoretical background of polarization states of light is given. In Section 3, measurements of water IOPs and particulate VSFs, and the instruments we used to measure polarized light fields, are reported. In Section 4, the numerical model we used for simulating the light fields is described. In Section 5, measured and simulated light fields under various conditions are shown and compared. In Section 6 , the measured and simulated DoLP and AoLP patterns are analyzed as a function of the scattering angle. In Section $\mathbf{7}$, conclusions are given.

\section{Theoretical Background}

The polarization state of any light field can be conveniently described by the Stokes vector $\mathbf{I}=[I, Q, U, V]^{T}$, where $I$ represents the beam radiance, $Q$ and $U$ correspond to the linear polarization states along the parallel-perpendicular and $\pm 45^{\circ}$ directions, respectively, and $V$ corresponds to the circular polarization states. All these parameters have units of $\mathrm{W} /\left(\mathrm{m}^{2} \mathrm{~nm} \mathrm{sr}\right)$. A commonly used indicator of the magnitude of the linear polarization state is the DoLP, defined as

$$
\operatorname{DoLP}=\frac{\sqrt{Q^{2}+U^{2}}}{I} .
$$

The e-vector orientation can be described by the AoLP $\chi$ :

$$
\tan (2 \chi)=\frac{U}{Q}
$$


The angle $\chi$ can be defined to vary from $-90^{\circ}$ to $90^{\circ}[20]$ or from $0^{\circ}$ to $180^{\circ}$ [16], where $\chi=0^{\circ}$ or $180^{\circ}$ means that the e-vector is in a reference plane, which is the meridian plane in this work, and $\chi= \pm 90^{\circ}$ means that the e-vector is perpendicular to the reference plane. In addition to the radiance, which describes how much energy is carried by the light field, the DoLP and the AoLP characterize how this energy is distributed in different linear polarization states.

Although the light source, i.e., the Sun, is unpolarized, the light field is usually highly linearly polarized in the underwater environment [21]. This polarization comes from scattering by molecules and aerosols in the atmosphere, molecular and hydrosol scattering in the ocean, as well as Fresnel refraction through the air-sea interface. In the water, the polarization features are dominated by scattering off hydrosols, i.e., suspensions of inorganic and/or organic suspended particles. There are some exceptions where neutral points exist. Neutral points are specific directions where the DoLP goes to zero, which implies that the light field is unpolarized along those directions. Characterization of the DoLP and AoLP in the underwater environment is crucial for understanding how animals with linear polarization vision view their environment.

\section{Optical Instruments and Methods}

Measurements were acquired during a field experiment in June 2010, near Port Aransas, Texas, in the Gulf of Mexico. In the field experiment, absorption $\left(a_{\mathrm{pg}}, \mathrm{m}^{-1}\right)$ and attenuation $\left(c_{\mathrm{pg}}, \mathrm{m}^{-1}\right)$ coefficients were measured at nine visible wavelengths (ranging from 412 to $715 \mathrm{~nm}$ ) using a WET Labs ac-9 device. The effects of pure seawater were removed through the calibration procedure. Methods used for ac-9 purified water calibrations and corrections for temperature and salinity absorption dependence are described in Twardowski et al. [22]. Scattering errors in the ac-9 absorption channel were corrected using a method that convolved concurrently measured volume scattering functions (see below) with a weighting function describing the scattering error of the ac-9 reflective flow tube used for the absorption channel [23]. The volume scattering function $(\beta(\theta)$, $\mathrm{m}^{-1} \mathrm{sr}^{-1}$ ) was measured with a custom device called the Multi-Angle SCattering Optical Tool (MASCOT) [24]. The MASCOT measures $\beta(\theta)$ for monochromatic light $(658 \mathrm{~nm})$ between $10^{\circ}$ and $170^{\circ}$ at $10^{\circ}$ intervals, sampling at $20 \mathrm{~Hz}$. The MASCOT source beam is a $30 \mathrm{~mW}$ laser diode expanded with a Gallilean $2 \times$ beam expander, and is transmitted through a wedge depolarizer to provide the unpolarized light required to measure $\beta(\theta)$.

Discrete surface seawater samples were collected at each station and filtered onto Whatman GFF (nominal pore size $0.7 \mu \mathrm{m}$ ) filters. Filter pads were stored on dry ice while at sea, and then transferred to a $-80^{\circ} \mathrm{C}$ freezer. Chlorophyll $a$ was determined using high pressure liquid chromatography.
Two instruments were used to take measurements of the underwater polarized light field. A hyperspectral and multiangular polarimeter, developed by the Optical Remote Sensing Laboratory at the City College of New York (hereafter referred to as the "CCNY polarimeter"), measured the polarized light field at 136 wavelengths ranging from ultraviolet $(349 \mathrm{~nm})$ to infrared $(801 \mathrm{~nm})$. The hyperspectral radiance sensors (HyperOCRs, Satlantic) have a linear polarizer (Edmund Optics) in front of each sensor's window. The orientations of the polarizers were $0^{\circ}, 90^{\circ}$, and $45^{\circ}$ with respect to a reference axis. The radiometers were mounted on a scanning system controlled by an underwater electric stepper motor (Newmark Systems Inc.). Details of this instrument have been reported by Tonizzo et al. [14]. Figure 1(a) shows the CCNY polarimeter deployed with buoys to sample near-surface waters during the field campaign. Coincident downwelling irradiance was measured by an irradiance sensor (HyperOCR, Satlantic) mounted in an unobstructed and elevated position on the research vessel.

The University of Texas developed a prototype full Stokes vector video polarimeter (hereafter referred to as the "UT video polarimeter") to make underwater polarization measurements. The video polarimeter employed electronically controlled birefringence devices, called liquid crystal pi cells, in front of a conventional color video camera with red, green, and blue (RGB) wavebands. Two pi cells were used, rotated $22.5^{\circ}$ from each other, combined with a linear polarizer to measure four different polarization states. The four different polarization states were recorded on four consecutive frames of a progressively scanned camera at $60 \mathrm{fps}$. The resulting Stokes parameter images were calculated from the collective information stored in these four images. Because of the consecutive frame measurements, the resulting Stokes vector calculation was integrated over $67 \mathrm{~ms}$. The video polarimeter was calibrated by sampling the polarization state of linear polarizers in front of an illuminated unpolarized background in a diverse arrangement of polarization angles and intensity states. The video polarimeter was calibrated only to measure the normalized Stokes vector and does not give calibrated measurements of the intensity. An underwater housing unit allowed the polarimeter to make measurements of the ocean light field polarization [Fig. 1(b)]. Angular information on the oil rig measurements (near which experiments were made) were estimated by the geometrical relationships of the camera with the oil rig and the Sun. Unlike the CCNY polarimeter, the UT video polarimeter worked at a limited spectral range (RGB) and only results from the green channel $(\sim 510 \mathrm{~nm})$ are presented. While this prototype version will provide useful qualitative comparisons to the CCNY polarimeter (a point detector) and model simulations, near-future inclusion of position sensors will provide more accurate measurements for model simulations. 

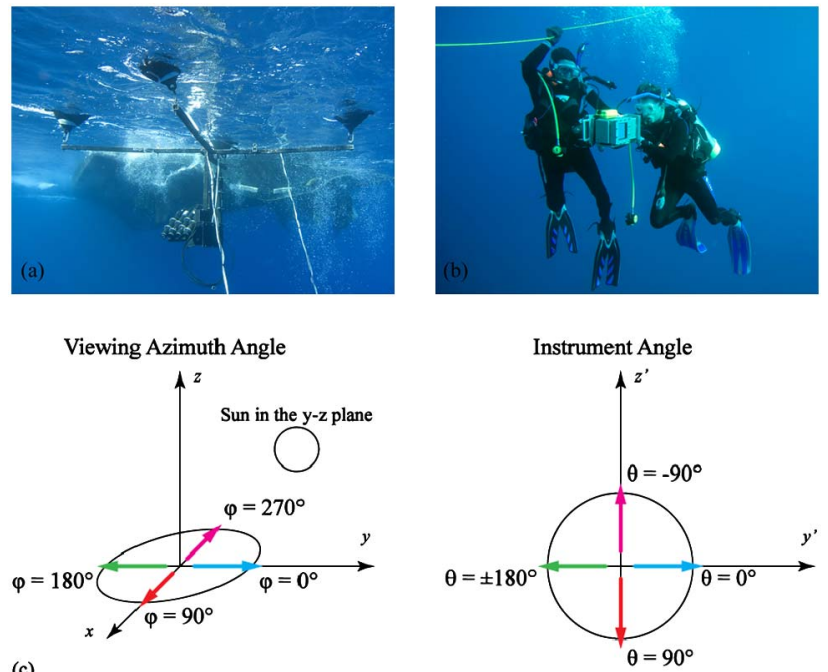

(c)

Fig. 1. (Color online) Photos of the two polarization instruments during deployment in the clear oceanic stations: (a) CCNY polarimeter taking near-surface measurements and (b) diver-operated UT video polarimeter. (Photos by Erich Schlegel.) (c) Viewing geometries for both polarimeters (arrows indicate instrument orientations).

\section{Monte Carlo Radiative Transfer Model}

Radiative transfer simulations were performed to generate the polarized light field that could then be compared with the measurements. We used a vector Monte Carlo model for a plane-parallel, multilayer coupled atmosphere-ocean system. The Monte Carlo algorithm used in the study is the same as that reported by Zhai et al. [17]. The incident solar irradiance spectrum was interpolated from tabulated values in Liou ([25], p. 56). The atmosphere-ocean system was modeled as follows: the atmosphere was modeled as a molecular Rayleigh scattering layer on top of an aerosol layer. The molecule layer is described by Rayleigh scattering, while the aerosol layer is governed by an oceanic aerosol model [26]. Both layers were assumed to be conservative [the atmospheric absorption at interested wavelengths was found to be negligible according to calculations using the line-by-line radiative transfer model (LBLRTM) [27]]. The wind-roughened ocean surface was modeled by the Cox and Munk [28] wave slope model. The ocean was simulated by a two-component model, with one component being water molecules and another being particles. The scattering and absorption spectra of pure sea water are provided by Zhang $e t$ al. [29] and by Pope and Fry [30], respectively; those of hydrosol particles and colored dissolved organic matter (CDOM) were from ac-9 measurements.

In this study, we made the best use of available field data to construct the particulate phase matrix. The VSFs were based on MASCOT measurements, as described in Section 5. The particulate phase matrix was based on a reduced Rayleigh phase matrix with a depolarization factor $\rho$ (see, for example, Adams et al. [19] and van de Hulst [31]) in the following form:

$$
\begin{aligned}
S_{12} / S_{11} & =\frac{(1-\rho)\left(\mu^{2}-1\right)}{\left(1+\mu^{2}\right)+\left(3-\mu^{2}\right) \rho}, \\
S_{22} / S_{11} & =\frac{(1-\rho)\left(\mu^{2}+1\right)}{\left(1+\mu^{2}\right)+\left(3-\mu^{2}\right) \rho}, \\
S_{33} / S_{11} & =\frac{2(1-\rho) \mu}{\left(1+\mu^{2}\right)+\left(3-\mu^{2}\right) \rho}, \\
S_{44} / S_{11} & =\frac{2(1-3 \rho) \mu}{\left(1+\mu^{2}\right)+\left(3-\mu^{2}\right) \rho} .
\end{aligned}
$$

Note that there are two definitions of the depolarization factor. Adams et al.'s definition of $\rho$ is equivalent to the quantity $\gamma$ in Chandrasekhar [32] (p. 80), and is related to Chandrasekhar's definition of $\rho_{n}$ as $\rho_{n}=$ $2 \rho /(1+\rho)$. The two definitions and their relation were discussed in great detail in van de Hulst [31]. In this study, we used $\rho=0.0588$ or $\rho_{n}=0.1 \overline{111}$, which corresponds to a maximum $-S_{12} / S_{11}$ of 0.8 . This phase matrix was then modified such that the peak in $-S_{12} / S_{11}$ shifts from $90^{\circ}$ to $94^{\circ}$. This $S_{12} / S_{11}$ pattern, including the maximum value and its location, has been observed in multiple measurements in clear waters, such as the Ligurian Sea and waters south of Hawaii using the MASCOT instrument (M. Twardowski, unpublished data). To measure the $-S_{12} / S_{11}$ element, routine switching of horizontal and vertical polarization filters in front of the unpolarized laser source beam controls the polarization state of the MASCOT (for example, the horizontal and vertical filters produce incident Stokes vectors $[1,1,0,0]^{T}$ and $[1,-1,0,0]^{T}$, respectively). Thus, unpolarized, horizontally $(H)$ and vertically $(V)$ polarized VSFs are continually measured. The $-S_{12} / S_{11}$ element is calculated as $-S_{12} / S_{11}=-(H-V) /$ $(H+V)$. The maximum value of $-S_{12} / S_{11}$ used in this study is slightly higher than that measured by Voss and Fry [33] of 0.66. The ac-9 and MASCOT measurements used in the radiative transfer code were those closest to the recording times of the CCNY polarimeter and the UT video polarimeter and represent the average IOPs of the surface water column during the polarimeters' recording time.

The atmospheric Rayleigh optical thickness was determined by the equation in Hansen and Travis [34]; the aerosol optical thickness was given by remotely sensed data provided by the NASA MODerateresolution Imaging Spectroradiometer (MODIS) Aqua satellite in the Gulf of Mexico on the dates of measurement, i.e., 17 and 22 June 2010.

\section{Comparisons between Measured and Simulated Polarization States}

Polarized light field data and IOP measurements are presented from two stations (A and B) with coordinates $27^{\circ} 45^{\prime} 50.8^{\prime \prime} \mathrm{N}, 96^{\circ} 37^{\prime} 52.0^{\prime \prime} \mathrm{W}$, and $27^{\circ} 43^{\prime} 1.3^{\prime \prime} \mathrm{N}$, $96^{\circ} 34^{\prime} 17.0^{\prime \prime} \mathrm{W}$, near two separate oil rigs on 17 and 22 June 2010 [Fig. 2(a)]. Measured chlorophyll $a$ concentrations were 0.20 and $0.12 \mathrm{mg} / \mathrm{m}^{3}$, respectively. In addition, we provide in Fig. 2(a) monthly composite $4 \mathrm{~km}$ resolution chlorophyll $a$ imageries from the 

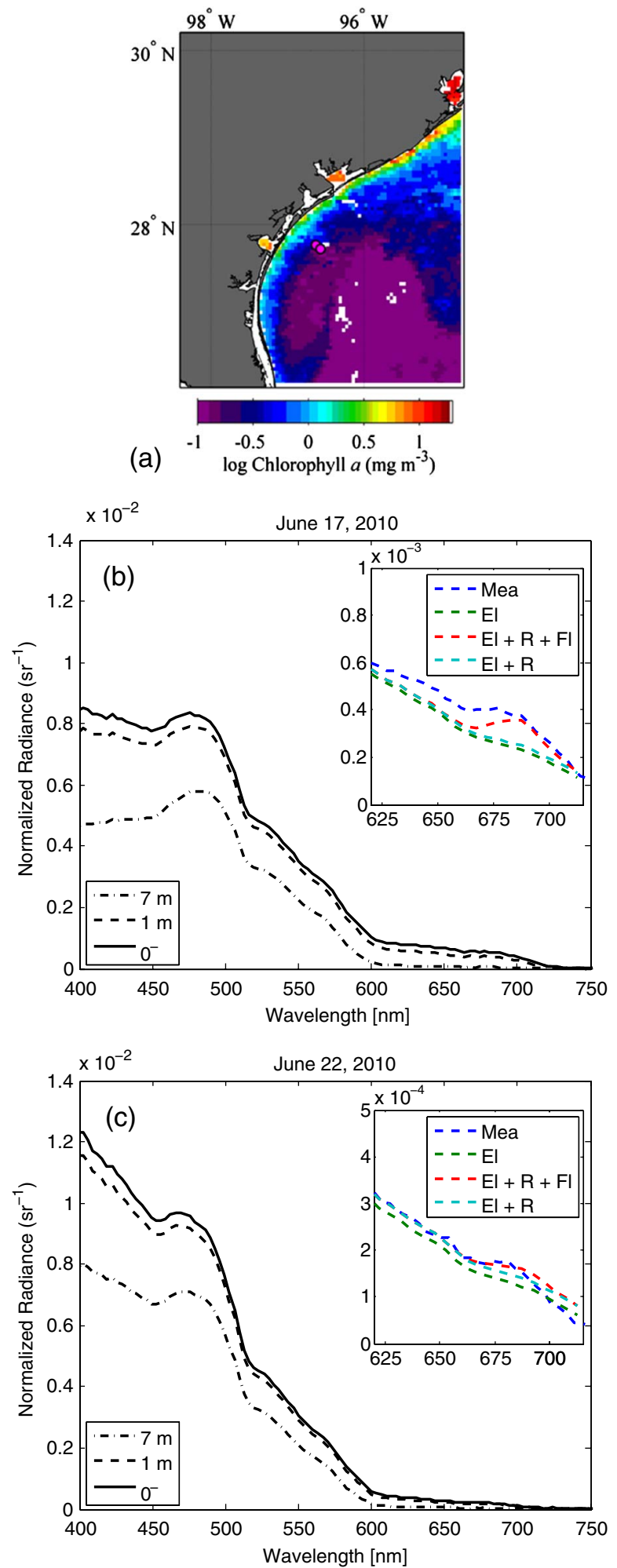

Fig. 2. (Color online) (a) Locations of the two field stations in the Gulf of Mexico (magenta circles) overlain on satellite-derived mean chlorophyll $a$ distributions for the month of June 2010 from the MODIS Aqua sensor. (b) and (c) Normalized radiance for the two locations at 7 and $1 \mathrm{~m}$ depth and extrapolated to the surface $\left(0^{-}\right)$. Inset in (b) and (c) is the normalized radiance spectra for the two locations at $1 \mathrm{~m}$, measured and simulated with Hydrolight (elastic and with Raman and chlorophyll fluorescence components).
MODIS Aqua sensor for the area of measurements in the Gulf of Mexico [35]. Normalized radiance spectra, calculated as upwelling nadir radiance normalized to above-water incident downwelling irradiance, measured at 1 and $7 \mathrm{~m}$ depth by the CCNY polarimeter [i.e., $L_{u}(z) / E_{d}\left(z=0^{+}\right)$], are shown in Figs. $2(\mathrm{~b})$ and 2(c), together with the values extrapolated to just beneath the sea surface (i.e., $0^{-}$) using the vertical downwelling diffuse attenuation coefficient estimated between 1 and $7 \mathrm{~m}$, which is considered a reasonable approach in these well-mixed waters. These radiance measurements agreed with independent radiance depth profiles collected with a HyperPro instrument (Satlantic, Canada; data not shown). In addition, measured normalized radiance spectra ("Mea") at $1 \mathrm{~m}$ depth for both stations are shown in Figs. $2(\mathrm{~b})$ and 2(c) as insets, together with simulated elastic spectra $(\overline{\mathrm{El}})$, the spectra including the elastic, Raman, and fluorescence components $(\mathrm{El}+R+\mathrm{Fl})$ and the spectra including the elastic and Raman components $(\mathrm{El}+R)$ using the Hydrolight radiative transfer program [36] . Fluorescence quantum yield at station $A$ was assumed as $2 \%$ and at station $B$ as $0.5 \%$. While the matches are not perfect, they give a basic estimation of the inelastic contributions to the total normalized radiance, which will be further used in the interpretation of the DoLP signals.

Water column depth was approximately $50 \mathrm{~m}$ at both locations. Measurements were made under different conditions and covered a wide range of solar elevations, viewing directions, and instrument depths (Table 1 ). Specifically, the listed solar elevations are mean values along with variations during a CCNY polarimeter measurement, which took about $15 \mathrm{~min}$. The measurements on 22 June correspond to a low sea state condition, with surface wind speeds lower than $1 \mathrm{~m} \mathrm{~s}^{-1}$, while the measurements on 17 June correspond to a higher sea state condition, with surface wind speeds $\sim 6 \mathrm{~m} \mathrm{~s}^{-1}$. Different station numbers (e.g., A1 or B3) correspond to different measurements obtained over time at the same location. The position of the research vessel remained constant during deployment at each station by tying up to the oil rigs.

Figure 3 shows the measured particulate and CDOM absorption and attenuation spectra $\left(a_{\mathrm{pg}}\right.$ and $c_{\mathrm{pg}}$, respectively) at the stations. For the IOP measurements, stations A1 and B1 were profiled from 1 to $20 \mathrm{~m}$. Station B4 was sampled only at two depths close to $4 \mathrm{~m}$. Figures 3(a) and 3(b) show the full spectra of the mean along with 1 standard deviation from the mean (error bars) measured in each profile. Figures 3(c)-3(f) show the vertical profiles for selected wavelengths measured at stations A1 and B1, respectively. Except for $a_{\mathrm{pg}}$ at station A1, the IOPs are relatively homogeneous with depth (the error bars are small), and exhibit little variability over the course of the day (the difference between B1 and B4 is small). However, as shown in Fig. 3 and according to the chlorophyll $a$ data, the 22 June measurements represent a slightly clearer water body than the 17 June measurements. 
Table 1. Relevant Parameters for the Two Stations

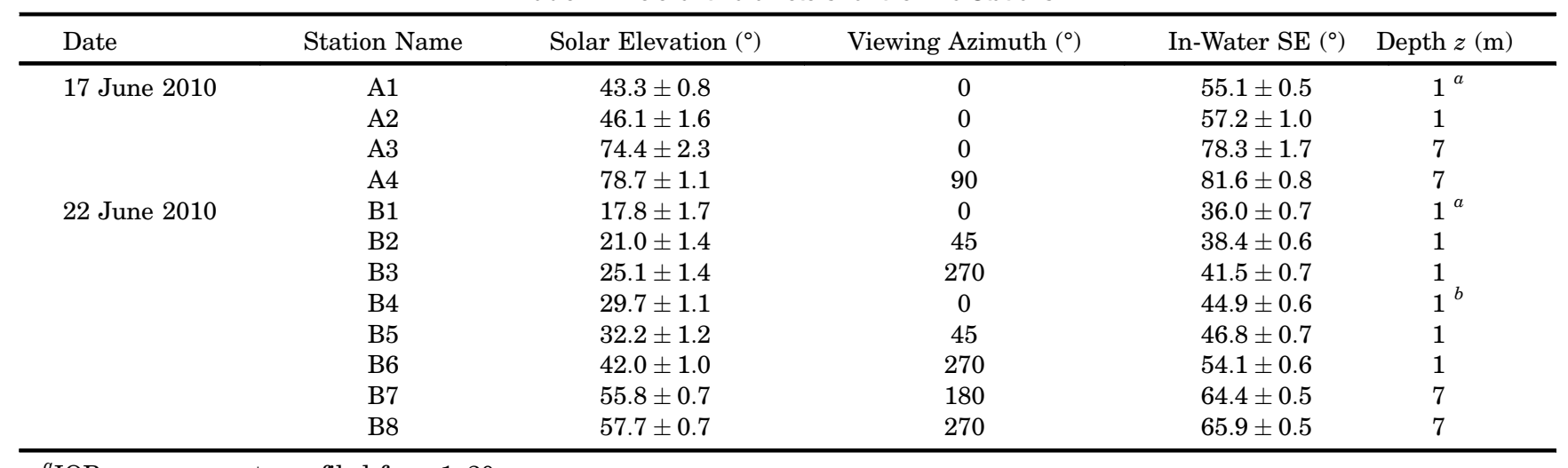

${ }^{a}$ IOP measurements profiled from $1-20 \mathrm{~m}$.

${ }^{b}$ IOP measurements taken at $\sim 4 \mathrm{~m}$.

Measurements of $\beta(\theta)$ using MASCOT were performed along with the IOP measurements at stations A1 and B1 (profiled from 1 to $20 \mathrm{~m}$ ) and station B4 (sampled only at a depth of $4 \mathrm{~m}$ ). The overall shapes of $\beta(\theta)$ at the stations were very similar [Fig. $\underline{4(\mathrm{a})}$ ].
Error bars represent 1 standard deviation from the mean obtained in the surface layer. Figure $4(\mathrm{~b})$ shows $\beta(\theta)$ measurements at station B1 along with the standard Petzold phase function [37] and a HenyeyGreenstein (H-G) phase function [38] with an
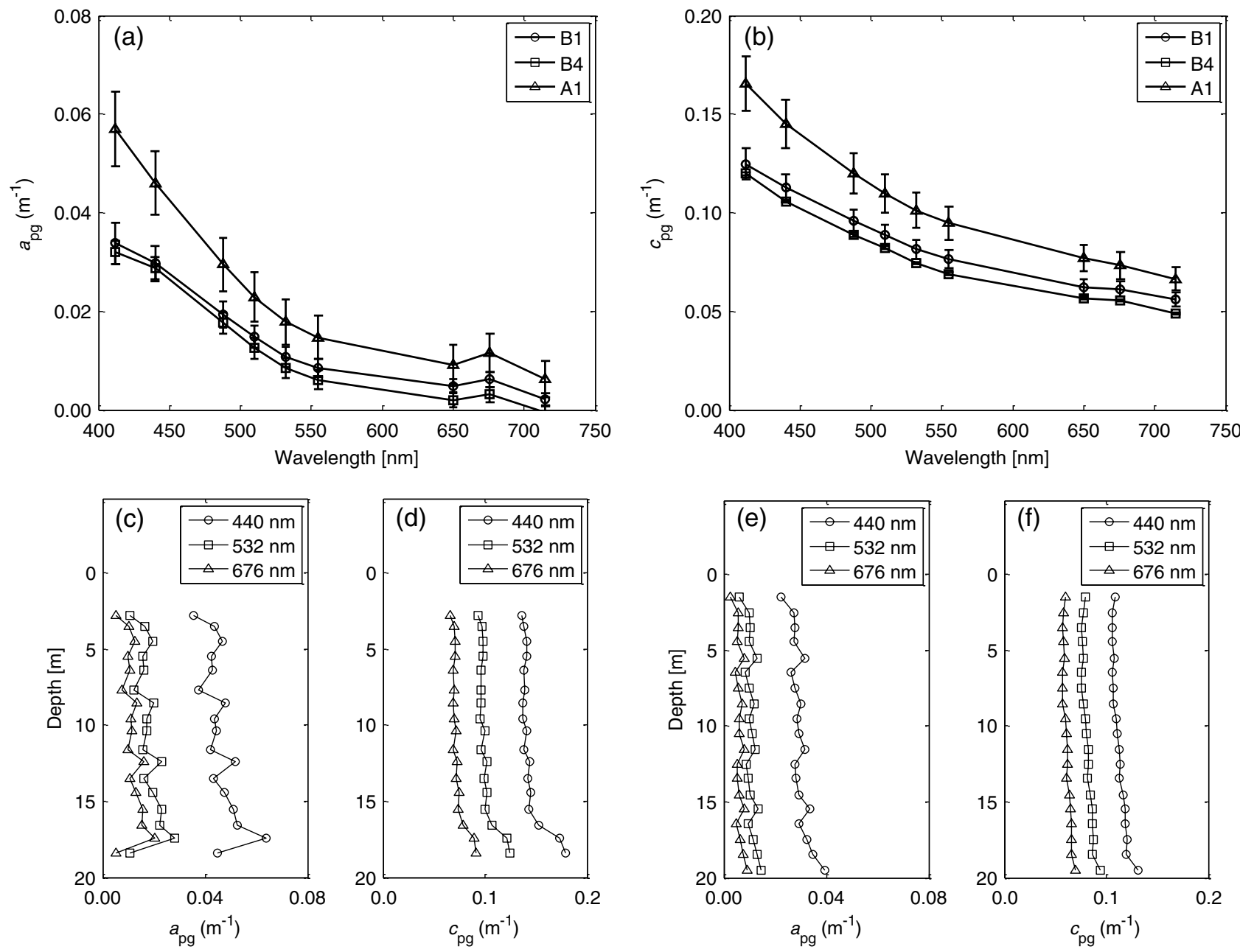

Fig. 3. (a) and (b) Particulate and CDOM absorption $\left(a_{\mathrm{pg}}\right)$ and attenuation $\left(c_{\mathrm{pg}}\right)$ spectra measured using the WET Labs ac-9-MASCOT package at stations B1, B4, and A1. Error bars represent 1 standard deviation of the mean value obtained during each measurement. (c) and (d) Vertical profiles of $a_{\mathrm{pg}}$ and $c_{\mathrm{pg}}$ for selected wavelengths at station A1. (e) and (f) Same as (c) and (d), but for station B1. 
asymmetry factor of $g=0.95$, both scaled to fit the forward-scattering measurement from the MASCOT. In a previous experiment in the coastal waters of New York Harbor [14], measured $\beta(\theta)$ were similar to the Petzold phase function. In these clearer open ocean waters, however, $\beta(\theta)$ are closer to this H-G phase function rather than the Petzold phase function. Therefore, to generate a full $\beta(\theta)$ from $0^{\circ}$ to $180^{\circ}$ that could be used in model simulations, we extended the MASCOT measurements in the forward direction using the forward peak from the H-G phase function (takes the $\mathrm{H}-\mathrm{G}$ phase function with $g=0.95$ from $0^{\circ}$ to $10^{\circ}$, rescales it such that the value at $10^{\circ}$ equals to the measured $\beta\left(10^{\circ}\right)$ ) and in the backward direction (from $170^{\circ}$ to $180^{\circ}$ ) by extrapolation. Since the main contribution to the scattered light comes from the small angle area of less than $10^{\circ}$, it is critical to check the validity of the extension to small angles. We compared the particulate scattering coefficient $\left(b_{p}\right)$ computed from these extended $\beta(\theta)$ functions with that derived from ac-9 measurements $\left(c_{\mathrm{pg}}-a_{\mathrm{pg}}\right.$ for $\left.b_{p}\right)$ using an acceptance angle of $0.90^{\circ}$ and found them to be consistent (Table 2).

We performed polarized Monte Carlo simulations for all stations listed in Table 1 , at all nine ac-9 wavelengths. In the next subsections, we present comparisons of the DoLP and AoLP, as well as normalized Stokes parameters $Q / I$ and $U / I$ between these simulations and the measured polarization states from both the CCNY polarimeter and the UT video polarimeter.

\section{A. Degree of Linear Polarization}

Figures 5(a)-5(i) show the comparison of the modelsimulated and measured DoLP patterns at all ac-9 wavelengths for station B1 (in the principal plane). The measurements were obtained from the CCNY polarimeter. Here the instrument angle varied from $-100^{\circ}$ to $150^{\circ}$ and is defined as follows: $-90^{\circ}$ is when the instrument looks upward, $90^{\circ}$ is when it looks
Table 2. Comparisons of the Particulate Scattering Coefficient $b_{p}$ Derived from the Extended VSFs and that Derived from ac-9 Measurements $\left(c_{\mathrm{pg}}-a_{\mathrm{pg}}\right.$ for $\left.b_{p}\right)$

\begin{tabular}{cccc}
\hline Station & VSF-Derived & ac-9-Derived & Difference $^{a}(\%)$ \\
\hline A1 & 0.07326 & 0.07066 & 3.68 \\
B1 & 0.05604 & 0.05782 & -3.08 \\
B4 & 0.05453 & 0.05262 & 3.63 \\
\hline
\end{tabular}

${ }^{a}$ The difference is defined as (VSF - ac-9)/ac-9.

downward, and $0^{\circ}$ is when it looks horizontally into the solar half plane, as shown in Fig. 1(c). One can see that the consistency between the measured and simulated data sets is excellent at all wavelengths less than $555 \mathrm{~nm}$. In both simulated and measured upwelling light fields (instrument angle greater than $\left.0^{\circ}\right)$, the maximum value in the DoLP at instrument angles around $49^{\circ}$ (i.e., scattering angle around $94^{\circ}$ ) increases from about 0.45 to about 0.6 as the wavelength increases from 412 to $555 \mathrm{~nm}$. For instrument angles around $-45^{\circ}$, i.e., in the forward-scattering direction, the measurements have substantial variations at all wavelengths due to the influence of surface waves [see Figs. $5(\mathrm{j})-5(\mathrm{r})]$. The measurements become very noisy at the red wavelengths (650, 676 , and $715 \mathrm{~nm}$ ) for the whole angular range due to low signals. However, it can still be seen that, at 650 and $676 \mathrm{~nm}$, the measured DoLP is slightly lower than the simulations. This is due to chlorophyll fluorescence [14] and Raman scattering [39] at these wavelengths, which the Monte Carlo radiative transfer model did not consider.

To view the comparison of the DoLP spectra in a compact way, in Fig. 6 we show the DoLP spectra at three relevant instrument angles for station B2. Full spectra of measured DoLP (smoothed to minimize noise effect) are presented and compared with multispectral simulations. Again, the consistency at all presented instrument angles is very good at blue
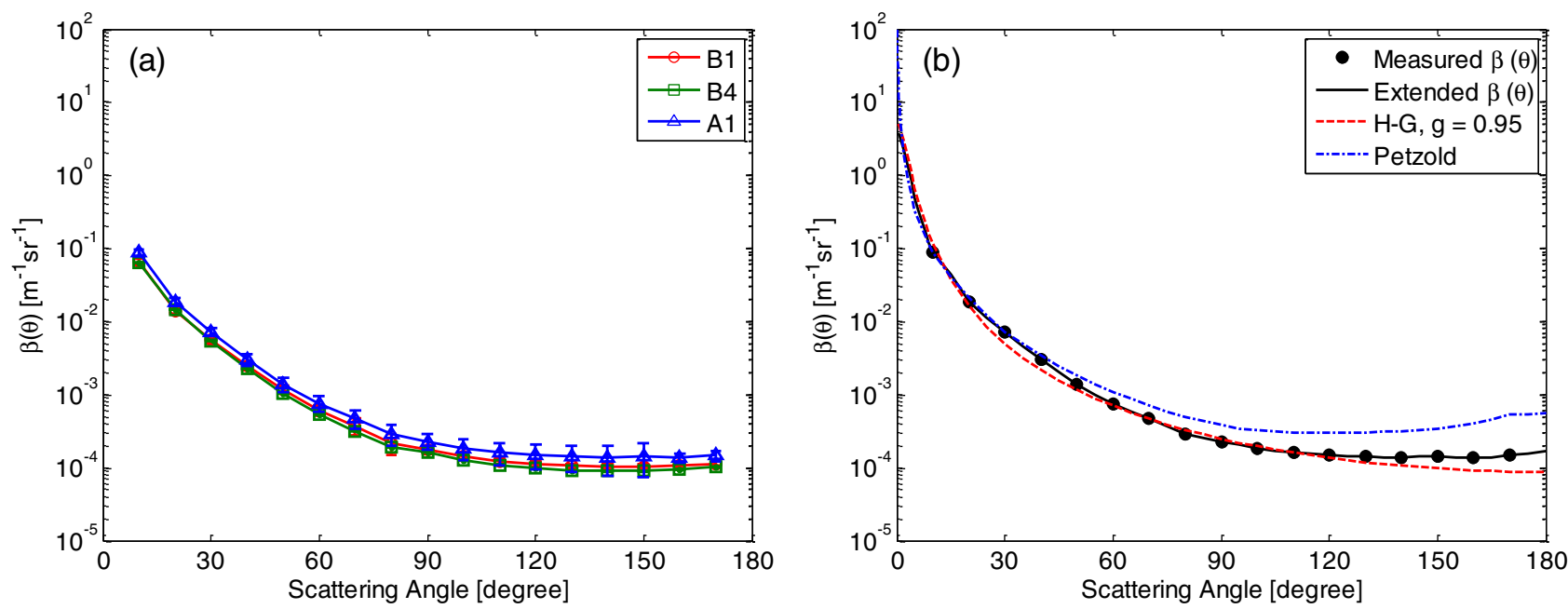

Fig. 4. (Color online) (a) $\beta(\theta)$ measured at selected stations using the MASCOT. Error bars show vertical variations. (b) Comparison of the MASCOT-measured $\beta(\theta)$, a $0^{\circ}$ to $180^{\circ}$ extrapolated $\beta(\theta)$ function based on the MASCOT measurement, a scaled H-G phase function, and a scaled Petzold phase function. 


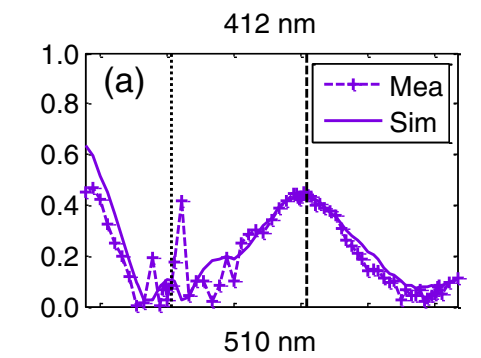

$\mathrm{B} 1 ; \mathrm{SE}=17.8 ; \mathrm{VA}=0 ; z=1 \mathrm{~m}$
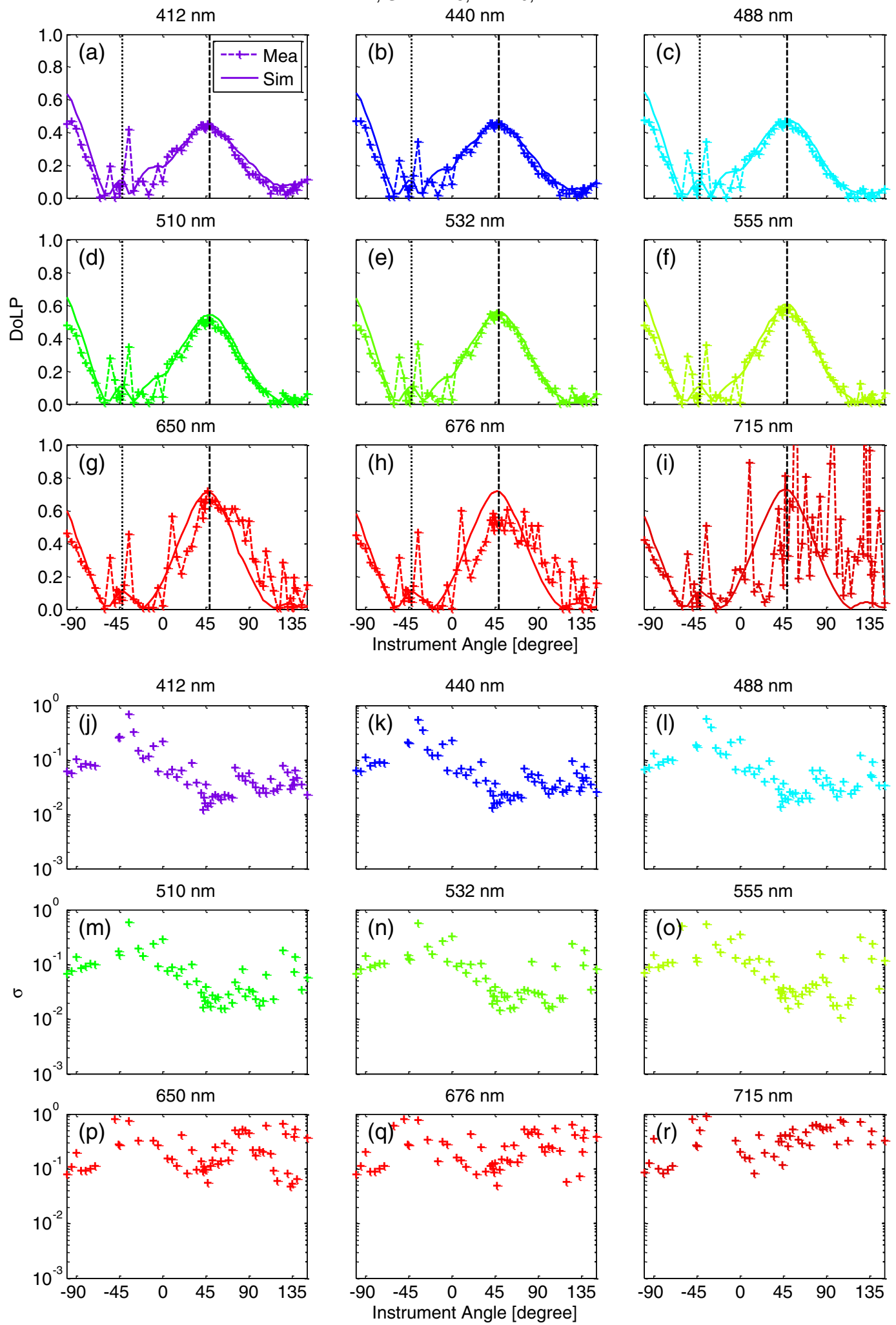

Fig. 5. (Color online) (a)-(i) DoLP patterns from CCNY measurements and from simulations at ac-9 wavelengths for station B1; the vertical dashed line indicates the angle that corresponds to a $94^{\circ}$ scattering angle; the vertical dotted line indicates the edge of Snell's window, with angles within the window to its left. Details of this station can be found in Table 1 and are listed in the figure title for convenience, where SE stands for solar elevation, VA stands for viewing azimuth, and $z$ is the detector depth. (j)-(r) Standard deviations for the DoLP measurements at ac-9 wavelengths for station B1. 
and green wavelengths (up to $600 \mathrm{~nm}$ ). At red wavelengths $(650 \mathrm{~nm}$ and longer), the data become noisy, but the influence of chlorophyll fluorescence and Raman scattering is still noticeable (decrease of the DoLP near $685 \mathrm{~nm}$ at $45^{\circ}$ in Fig. 6; this decrease was not observed at the other two instrument angles since the DoLP there was very low and noise dominates). We recently showed [40] that the relative contribution of fluorescence to the total reflectance is equal to the ratio of the DoLP dip due to the fluorescence and the elastic DoLP. This is roughly confirmed by the comparison of the inset in Fig. 2(c) for the relative fluorescence contribution and Fig. 6 for the relative DoLP dip due to the fluorescence where both are about $25 \%-30 \%$.

Next we focus on the DoLP pattern at one wavelength, $510 \mathrm{~nm}$, and study its dependence on the solar elevation and viewing azimuth angles. Figure 7 shows comparisons of DoLP patterns at the six B stations from simulations and from measurements by the CCNY polarimeter. These stations correspond to a low wind and clear water situation. The water IOPs including particulate VSFs are similar, and the instrument depth is $1 \mathrm{~m}$ for all stations. Therefore, these results show the dependence of the DoLP pattern on the solar elevation and viewing azimuth angles. Solar elevation increases from $17.8^{\circ}$ to $42.0^{\circ}$ sequentially through the panels. The left, middle, and right panels show results in the principal plane (viewing azimuth $=0^{\circ}$ ), and $45^{\circ}$ from and perpendicular to the principal plane, respectively. The comparison in the perpendicular plane is shown only for instrument angles of less than $65^{\circ}$; beyond this point, data are not reliable due to an instrumentation error that comes from the shadowing by one of the cables on one of the sensors (a sharp decrease in the intensity appears in this sensor's reading after

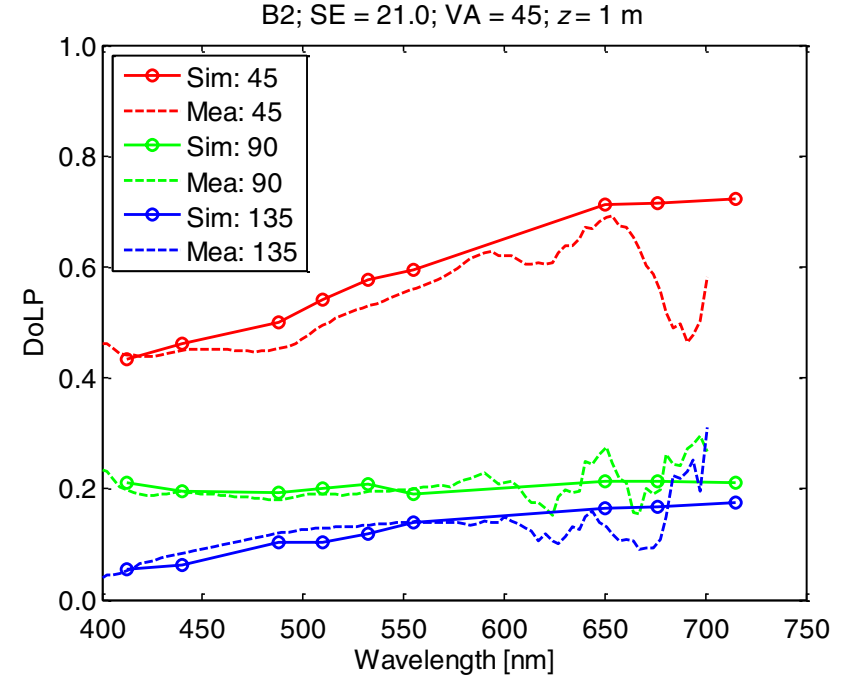

Fig. 6. (Color online) DoLP spectra from simulations (solid curves) and from CCNY polarimeter measurements (dashed curves) at relevant instrument angles for station B2. Measured data points at $715 \mathrm{~nm}$ for station B2 are not shown due to substantial uncertainties in this case.

$65^{\circ}$; data not shown). First of all, the overall consistencies in the $0^{\circ}$ and $45^{\circ}$ meridian planes are very good throughout the entire instrument angle range. Some minor discrepancies include, in the $45^{\circ}$ meridian plane, (1) the simulated DoLP is slightly higher from instrument angles $-30^{\circ}$ to $0^{\circ}$, and (2) the simulated DoLP peak is slightly shifted to larger instrument angles. In the perpendicular plane, the consistency between measurements and simulations inside Snell's window (instrument angles from $-90^{\circ}$ to $-45^{\circ}$ ) is relatively poor. Finally, although the maximum DoLP increases as the solar elevation increases in the principal plane and the $45^{\circ}$ meridian
$510 \mathrm{~nm}$
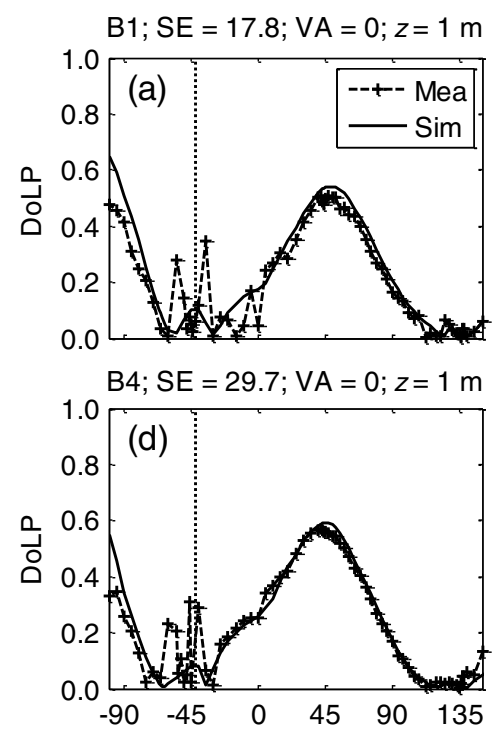

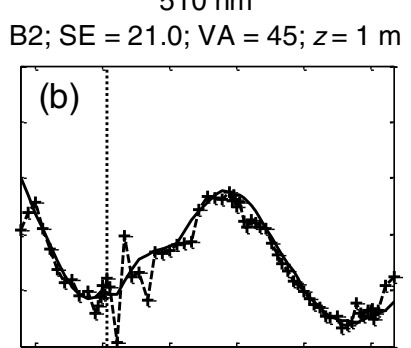

$\mathrm{B} 5 ; \mathrm{SE}=32.2 ; \mathrm{VA}=45 ; z=1 \mathrm{~m}$

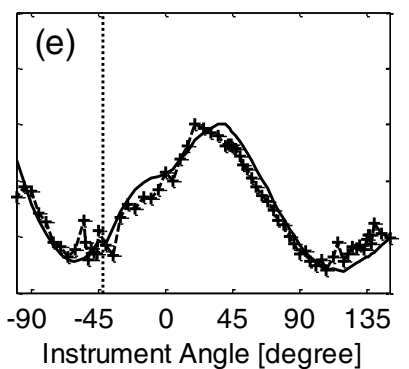

$\mathrm{B} 3 ; \mathrm{SE}=25.1 ; \mathrm{VA}=270 ; z=1 \mathrm{~m}$

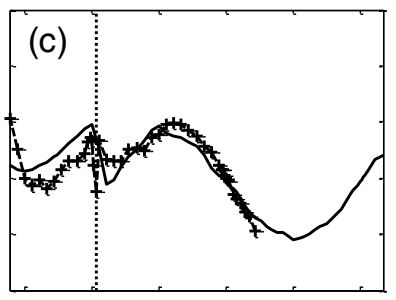

$B 6 ; \mathrm{SE}=42.0 ; \mathrm{VA}=270 ; z=1 \mathrm{~m}$

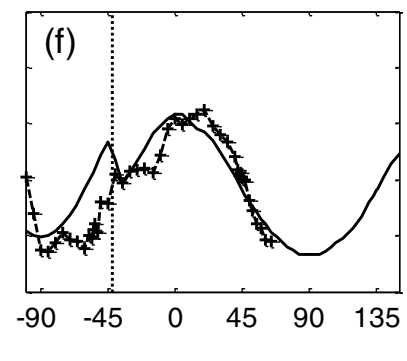

Fig. 7. Same as Figs. $5(\mathrm{a})-5(\mathrm{i})$, but with a fixed wavelength $(510 \mathrm{~nm})$ and various combinations of SE and VA angles. The water IOPs and VSFs were similar for all six stations. 
plane, the dependence of the DoLP pattern on the solar elevation is not obvious. This dependence can be better studied by looking at simulation results, where we can vary the solar elevation in a wider range (see Fig. 19 in Section 6). In the perpendicular plane, the DoL $\overline{\mathrm{P}}$ in Snell's window is substantially lower when the solar elevation is higher. The above discussions apply to all blue and green wavelengths. In the red, the agreement in the perpendicular plane was generally poorer than observed for the rest of the visible spectrum.

In Figs. 8(a) and 8(b), we show the DoLP patterns at stations $\overline{\mathrm{B} 7}$ and $\overline{\mathrm{B} 8}$, which are at $7 \mathrm{~m}$ below the ocean surface, with a higher solar elevation, and in the principal plane and perpendicular to the principal plane, respectively. Data in Fig. 8(a) at instrument angles greater than $120^{\circ}$ and those in Fig. 8(b) greater than $75^{\circ}$ are not shown (data are not reliable due to the instrument error mentioned above). It should be noted that Fig. 8(a) shows the other half of the principal plane (away from the Sun, viewing azimuth angle $\mathrm{VA}=180^{\circ}$ ), where a double-peak feature can be found in both measured and simulated data, although the height of one peak is not the same for measured and simulated data. The peak to the right is outside Snell's window and is due to a combination of the internal reflection and in-water scattering; the peak to the left is in Snell's window and comes from a peak in the polarized skylight. In the perpendicular plane, the two halves of the meridian plane are symmetric with respect to the instrument angle $90^{\circ}$. In this case, the DoLP patterns at $1 \mathrm{~m}$ [Fig. 7(f)] and $7 \mathrm{~m}$ [Fig. 8(b)] are similar.

Figures 8(c)-8(f) show the DoLP patterns for the A stations, which correspond to a slightly more turbid water body and a higher sea state condition (wind speed of about $6 \mathrm{~m} \mathrm{~s}^{-1}$ ). Shown are results in the principal plane [Figs. 8(c)-8(e)] and in the $\mathrm{VA}=270^{\circ}$ plane [Fig. 8(f)], with the solar elevation increasing from $43.3^{\circ}$ to $78.7^{\circ}$. The detector depth is still $1 \mathrm{~m}$ below the surface for stations A1 and A2 and $7 \mathrm{~m}$ for stations A3 and A4. In all four cases, the consistency in the angular range from around $-40^{\circ}$ to around $90^{\circ}$ is very good. Specifically, when the solar elevation increases from $43.3^{\circ}$ to $46.1^{\circ}$ (a less than $3^{\circ}$ increment in the solar elevation), the DoLP peak at around $40^{\circ}$ becomes narrower both in the simulated and in the measured data sets. For the higher solar elevation, the peak shifts to around a $20^{\circ}$ instrument angle, which still corresponds to a scattering angle of around $94^{\circ}$. Similar to the B stations, the measurements for the A stations are quite noisy and generally higher than the simulations in Snell's window. The measured DoLP at stations A1 and A2 is higher also in the angular range from $100^{\circ}$ to $135^{\circ}$. Despite higher sea state conditions, the agreement between measurements and simulations is quite good.

Now we turn to the measured data from the UT video polarimeter. Measurements were performed along with the CCNY polarimeter measurements for station B9, but deeper $(10 \mathrm{~m})$. The UT video polarimeter did not measure the angular distribution in a specific meridian plane. Instead, it measured polarization states along 37 representative viewing directions with combinations of various viewing azimuth and instrument angles. The measurement angles for the UT video polarimeter were determined by estimating the geometrical relationships of the Sun with various landmarks of the oil rig platform as viewed in the video data. Monte Carlo simulations were carried out using the same system setup (i.e., water IOPs, aerosol optical thickness) as aforementioned, but at $10 \mathrm{~m}$ and along the video polarimeter viewing directions. Since the video polarimeter works at the green wavelength, $510 \mathrm{~nm}$, simulations were performed only at this wavelength. Figure 9(a) shows the direct
$510 \mathrm{~nm}$

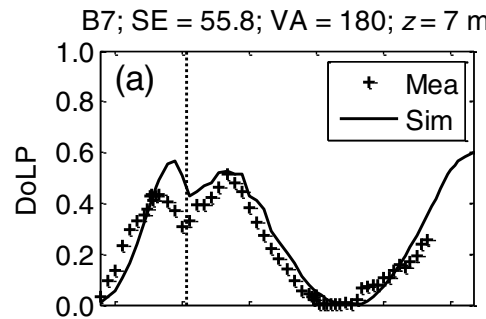

$\mathrm{A} 2 ; \mathrm{SE}=46.1 ; \mathrm{VA}=0 ; z=1 \mathrm{~m}$

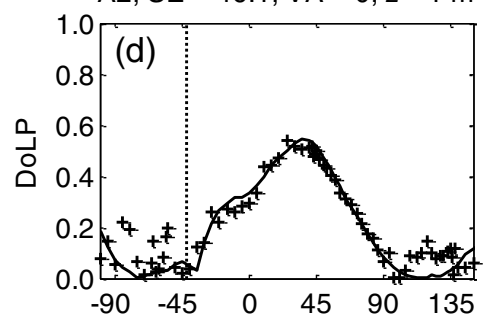

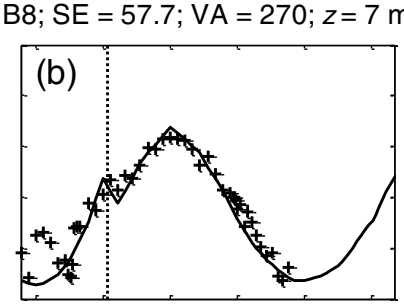

$\mathrm{A} 3 ; \mathrm{SE}=74.4 ; \mathrm{VA}=0 ; z=7 \mathrm{~m}$

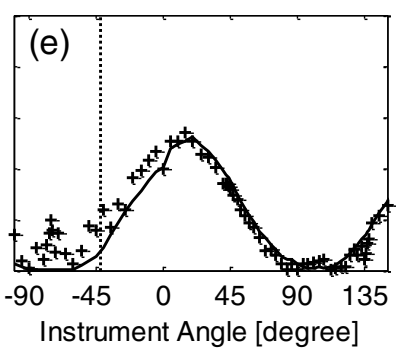

$\mathrm{A} 1 ; \mathrm{SE}=43.3 ; \mathrm{VA}=0 ; z=1 \mathrm{~m}$

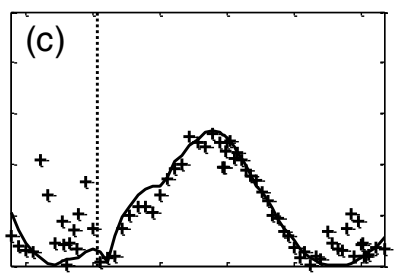

$\mathrm{A} 4 ; \mathrm{SE}=78.7 ; \mathrm{VA}=270 ; z=7 \mathrm{~m}$

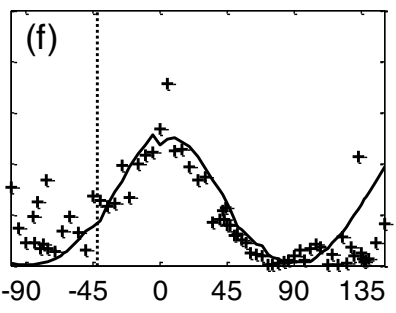

Fig. 8. Same as Fig. 7, but for stations B7 to B8 (larger depth) and A1 to A4 (slightly more turbid water body and higher sea state condition). 
comparison of the simulated and measured DoLP. While the correlation coefficient between the two data sets is relatively low $(r=0.58)$, this is to be expected with the qualitative nature of the prototype version of the UT video polarimeter. Figures 9(b)-9(d) present selected data points from the same data sets, but show the DoLP as a function of the viewing azimuth angle for three instrument angles: $-45^{\circ}, 0^{\circ}$, and $10^{\circ}$. The general trend of the azimuthal dependence predicted by the model is consistent with that in the measurements. However, there are several directions where measurements are considerably lower.

\section{B. Normalized Stokes Parameters $Q / I$ and $U / I$}

In Subsection 5.A, we see that the overall consistency between the measured and simulated DoLP patterns is excellent despite discrepancies in some cases. In this section, we try to find out where these discrepancies come from by showing the normalized Stokes parameters $Q / I$ and $U / I$.

Figures 10 (a) -10 (f) show the $Q / I$ and $U / I$ patterns corresponding to the DoLP patterns shown in Figs. 7(c), 7(e), and 7(f), i.e., stations B3, B5, and B6, respectively. Here we see that the agreements for both the $Q$ and $U$ components are generally quite good. However, at station B3 [Figs. 10(a) and 10(d)], we see that the substantial discrepancy in the DoLP measurement [Fig. 7(c)] from $-60^{\circ}$ to $45^{\circ}$ was mainly due to disagreement in the measured $Q / I$ component, while that from $-90^{\circ}$ to $-60^{\circ}$ was due to both components. At station B5 [Figs. 10(b) and 10(e)], the disagreement in the position of the DoLP peak [Fig. 7(e)] was mainly due to the $U / I$ component, while the higher DoLP measurement from $120^{\circ}$ to $150^{\circ}$ was due to the $Q / I$ component. At station $\mathrm{B} 6$ [Figs. $10($ c) and $10(\mathrm{f})$ ], the $U / I$ component was the main source for the discrepancy in the DoLP [Fig. 7(f)] from $-60^{\circ}$ and $-45^{\circ}$, as well as that in the position of the DoLP peak. For reference, the ambient radiance (I component) patterns are also shown in Figs. 10(g)-10(i). The measured and modelsimulated radiance patterns are generally consistent. The simulations are slightly lower than the measurements. This may come from the uncertainties in the aerosol model and/or the aerosol optical thickness.

Figure 11 is similar to Fig. 10 , but shows results for stations $\overline{\mathrm{A} 2}, \mathrm{~B} 7$, and $\mathrm{B} 8$. The measured $U$ component in the principal plane [Figs. 11(d) and 11(e)] is very noisy considering that it should be zero. However, this noisy $U$ component does not significantly affect the overall measured DoLP pattern [for example, at station A2, Fig. 8(d)] unless the $Q$ component is close to zero as well, in which case both the $Q$ and $U$ components contribute to give an overestimated DoLP [100 $0^{\circ}$ to $135^{\circ}$ in Fig. 8(d)]. This applies to measurements at stations $\mathrm{B} 1, \overline{\mathrm{B} 4}$, and $\mathrm{A} 1$, as well $(Q / I$ and $U / I$ patterns not shown). Similarly, at station B7, the lower measured DoLP [Fig. 8(a)] from $-60^{\circ}$ to $-45^{\circ}$ apparently comes from the lower $Q$ component measurement (note that the measured $U$ here is actually higher than simulation). An interesting case is the measurements at station B8, where the simulated and measured $Q / I$ and $U / I$ components [Figs. 11(c) and 11(f)] do not agree from $-50^{\circ}$ to $70^{\circ}$, while the DoLP pattern does [Fig. 8(b)].

From the above comparisons of measured and simulated $Q / I$ and $U / I$ patterns, it is clear that a disagreement in the DoLP can come from different sources: the $Q / I$ component only, the $U / I$ component only, or both components. Surprisingly, although the measured $U / I$ component is rather noisy in the principal plane, it does not necessarily introduce significant error in the DoLP pattern. Another lesson to learn is that, the measured and simulated $Q / I$ and $U / I$ components can be, different even if the DoLP patterns are consistent.
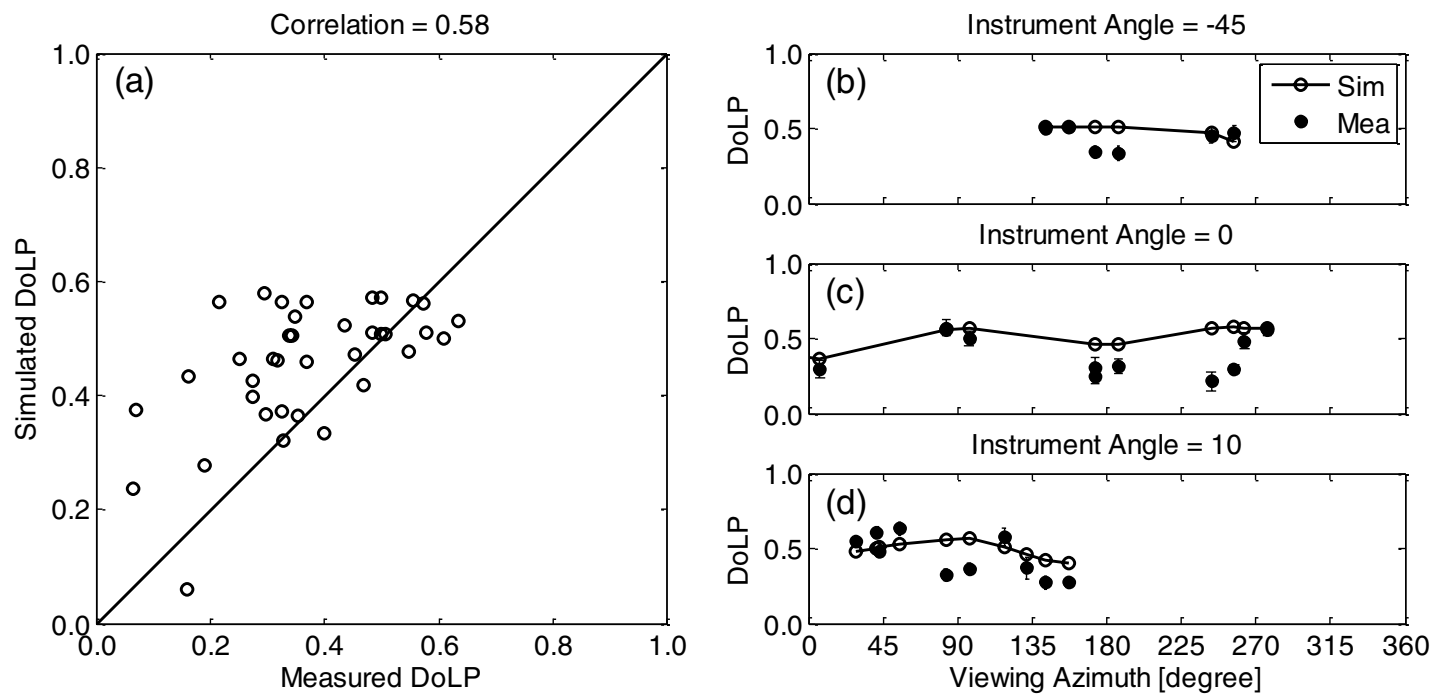

Fig. 9. (a) Direct comparison of DoLP at $10 \mathrm{~m}$, from UT video polarimeter measurements and from simulations. (b)-(d) Model-simulated and measured DoLP as a function of the viewing azimuth angle for selected instrument angles. 


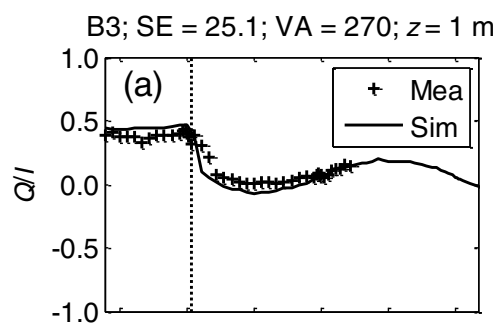

$\mathrm{B} 5 ; \mathrm{SE}=32.2 ; \mathrm{VA}=45 ; z=1 \mathrm{~m}$

$\mathrm{B} 6 ; \mathrm{SE}=42.0 ; \mathrm{VA}=270 ; z=1 \mathrm{~m}$
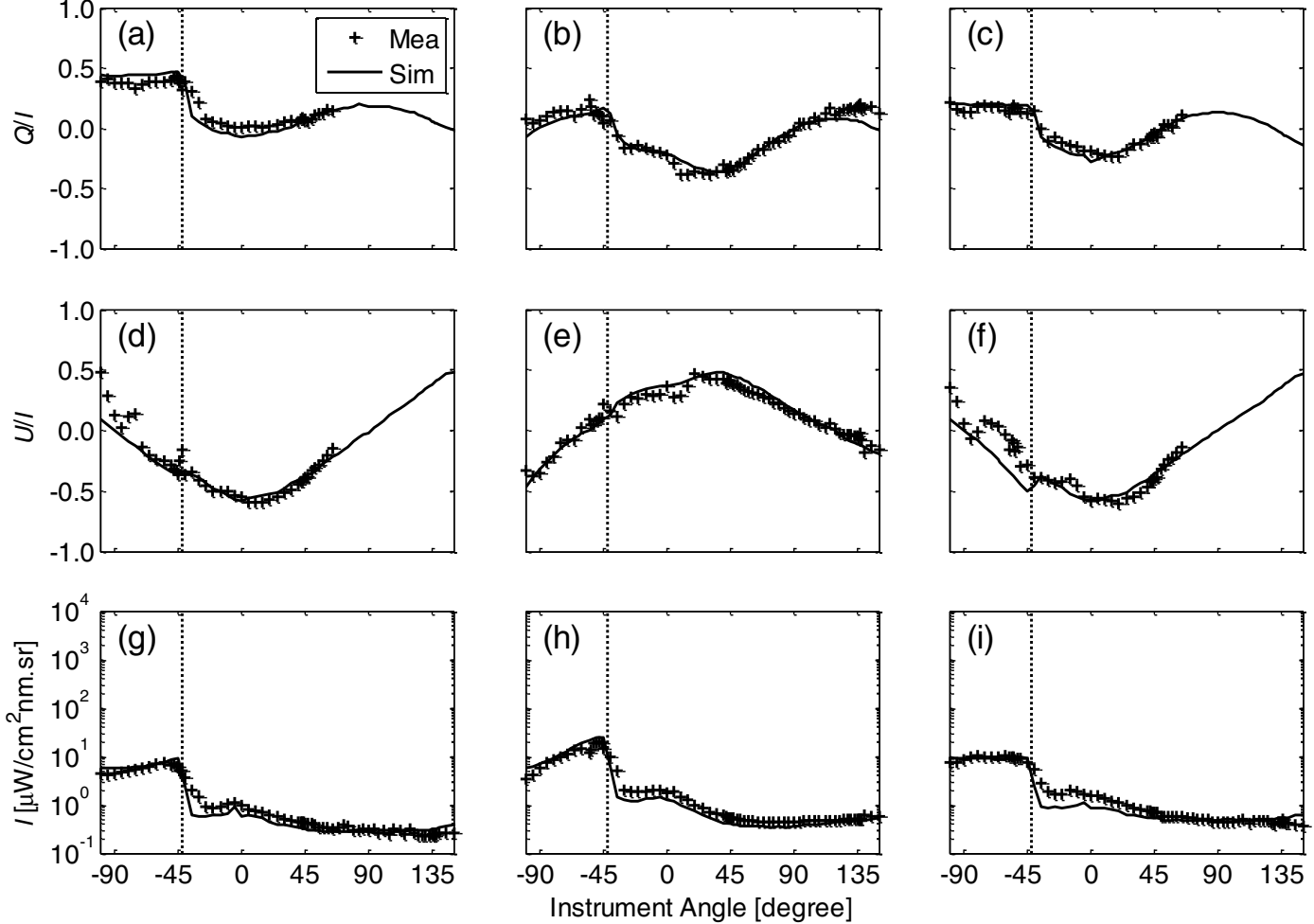

Fig. 10. Normalized Stokes components $Q / I$ (top panels) and $U / I$ (middle panels) from measurement and simulation at stations B3, B5, and B6. The radiance ( $I$ component) patterns are also shown in the bottom panels for reference.

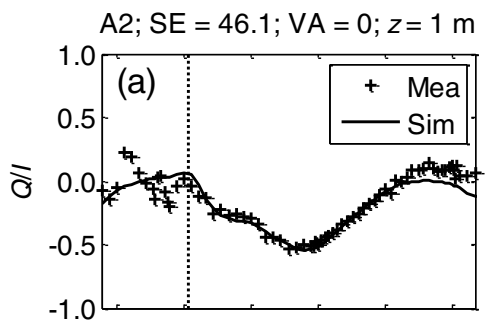

$\mathrm{B} 7 ; \mathrm{SE}=55.8 ; \mathrm{VA}=180 ; z=7 \mathrm{~m}$

$\mathrm{B} 8 ; \mathrm{SE}=57.7 ; \mathrm{VA}=270 ; z=7 \mathrm{~m}$
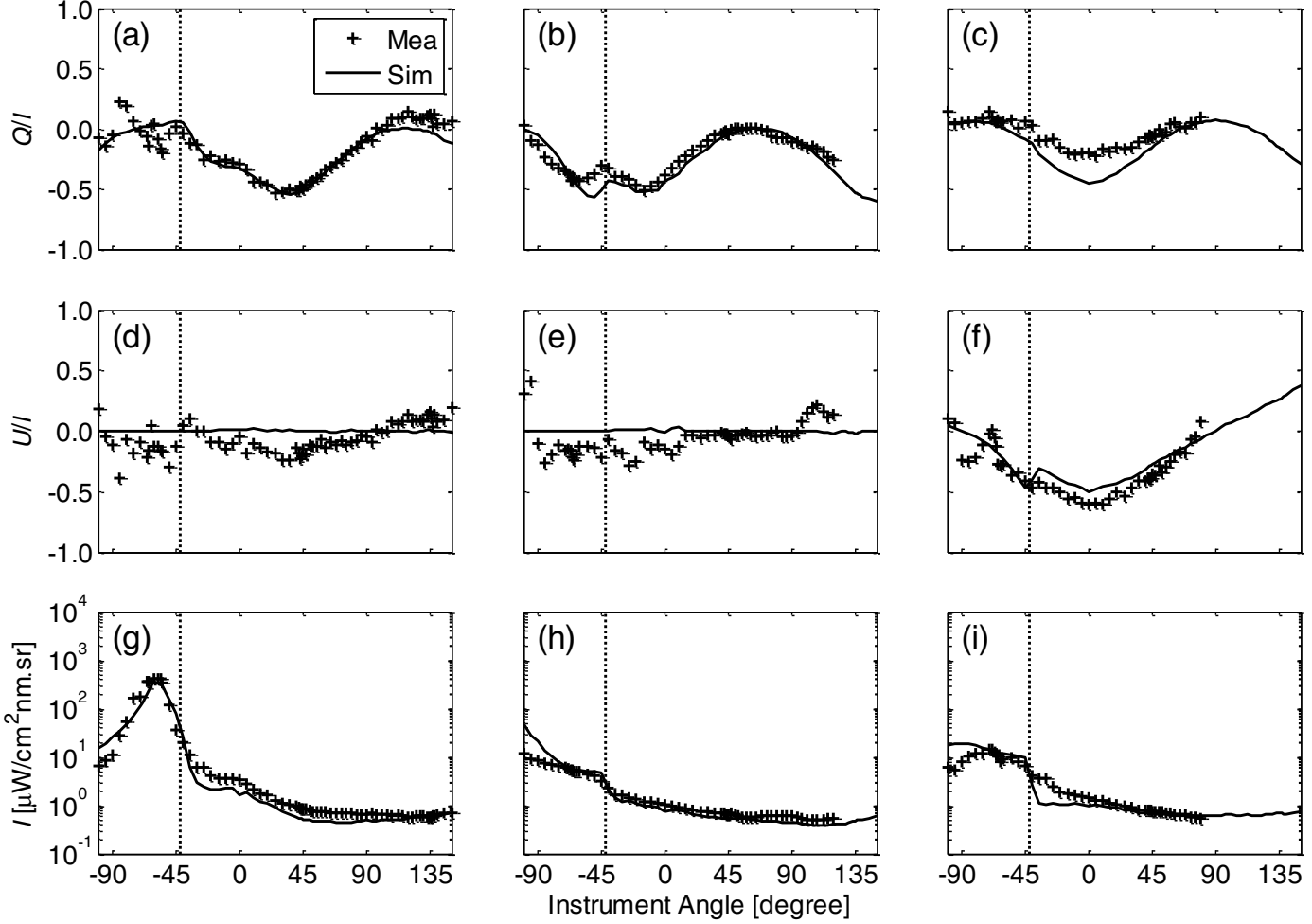

Fig. 11. Same as Fig. 10, but for stations A2, B7, and B8. 


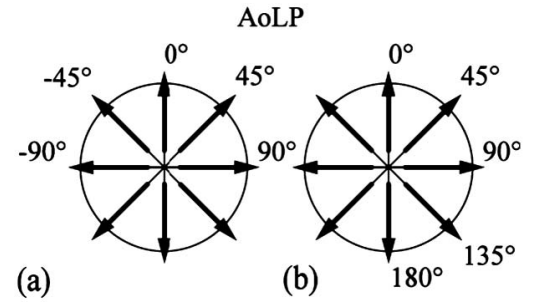

Fig. 12. Two definitions of the AoLP when looking into the direction of light propagation: (a) varying from $-90^{\circ}$ to $90^{\circ}$ [20]; (b) varying from $0^{\circ}$ to $180^{\circ}[16]$.

\section{Angle of Linear Polarization}

In this section we show the comparisons of the simulated and measured AoLP. Figure 12 shows two angular notations for the AoLP. As discussed, marine animals are sensitive to both DoLP and AoLP. Figure 13 is similar to Figs. 5(a)-5(i), but shows the comparison of measured and simulated AoLP at all nine wavelengths for station B2. Although the general pattern of AoLP does not vary much, there does exist a noticeable change in the shape of the pattern.

Shown in Fig. 14 are AoLPs from CCNY polarimeter measurements and from simulations for all stations out of the principal plane. In the principal plane, the AoLP is highly sensitive to the surface waves. We decided not to show those results even if, on average, the measured AoLP was close to $0^{\circ}$, as predicted by the theory. Figures $14(\mathrm{a})$ and $14(\mathrm{c})$ are results in the $45^{\circ}$ meridian plane at $1 \mathrm{~m}$ depth, Figs. 14(b) and 14(d) are results in the perpendicular plane at $1 \mathrm{~m}$ depth, and Fig. 14(e) is in the perpendicular plane but at $7 \mathrm{~m}$, all for low wind stations. Figure 14(f) is for a high wind station. Consistencies between simulated and measured AoLPs are found in most cases when the DoLPs agreed. One exception is for station B8 [Fig. 14(e)], where, from instrument angles $-50^{\circ}$ to $70^{\circ}$, the simulated and measured AoLPs show a $10^{\circ}$ discrepancy, while the DoLP [Fig. 8(b)] did agree. This implies that comparing the DoLP alone is not sufficient to guarantee that the full polarization states are consistent.

Additionally, in Fig. 15, we show the comparison of the simulated AoLP against measurements from the UT video polarimeter. In this comparison, quite a few data points are around $\pm 90^{\circ}$. To avoid the discontinuity at $\pm 90^{\circ}$, we used the AoLP definition shown in Fig. $13(\mathrm{~b})$, i.e., from $0^{\circ}$ to $180^{\circ}$. As shown in Fig. 15(a), unlike the poor correlation in DoLP, there is a fairly good consistency between the two sets of AoLPs, with a correlation coefficient $r$ of 0.79 . Figures 15(b)-15(d) show excellent agreement in the azimuthal dependence from model simulations and from video polarimeter measurements.

\section{Analyses of the Measured and Simulated Polarizations}

A simple interpretation can be given for polarized light fields in clear waters based on the polarization measurements available. It is well understood that,
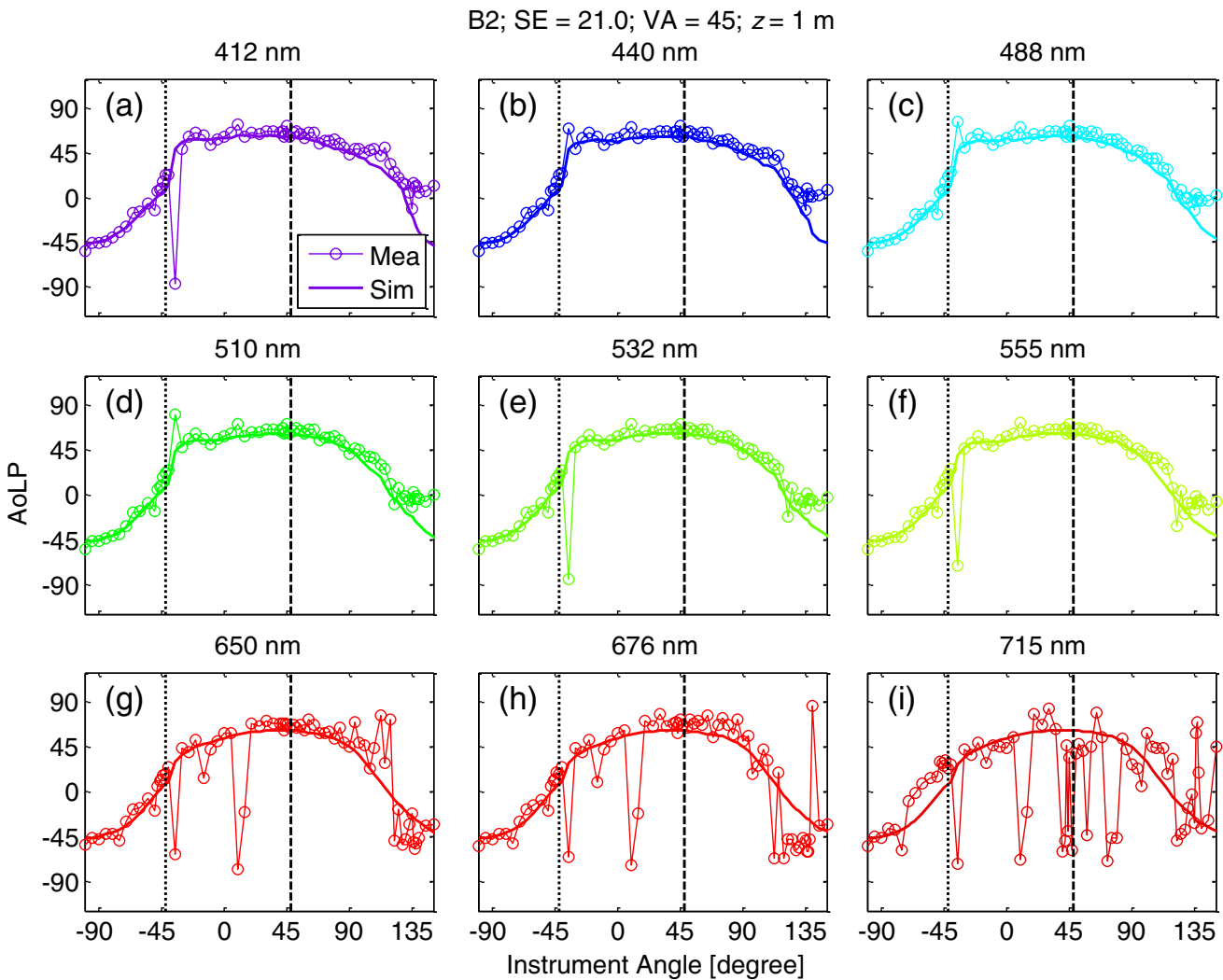

Fig. 13. (Color online) Similar to Figs. 5(a)-5(i), but showing the comparison of measured and simulated AoLP at all nine wavelengths for station B2. The AoLP is defined from $-90^{\circ}$ to $90^{\circ}$, as shown in Fig. 12(a). 
$\mathrm{B} 2 ; \mathrm{SE}=21.0 ; \mathrm{VA}=45 ; z=1 \mathrm{~m}$

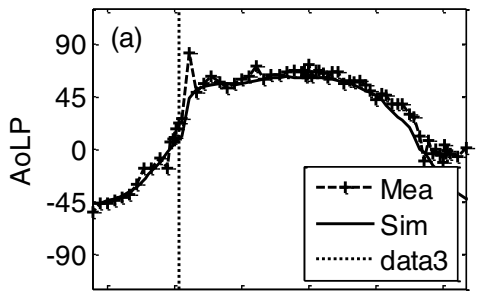

$\mathrm{B} 6 ; \mathrm{SE}=42.0 ; \mathrm{VA}=270 ; z=1 \mathrm{~m}$

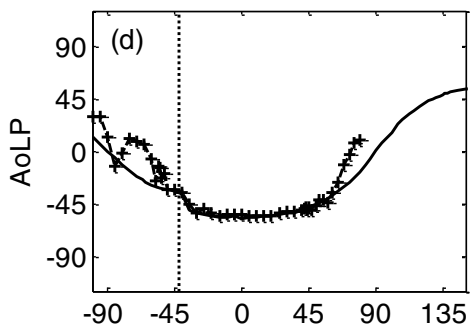

$\mathrm{B} 3 ; \mathrm{SE}=25.1 ; \mathrm{VA}=270 ; z=1 \mathrm{~m}$

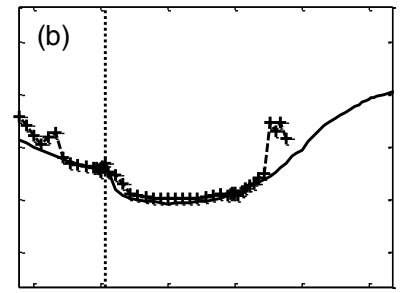

$\mathrm{B} 8 ; \mathrm{SE}=57.7 ; \mathrm{VA}=270 ; z=7 \mathrm{~m}$

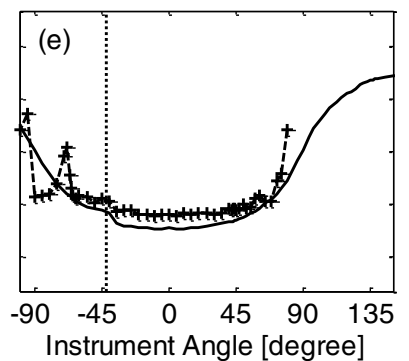

$\mathrm{B} 5 ; \mathrm{SE}=32.2 ; \mathrm{VA}=45 ; z=1 \mathrm{~m}$

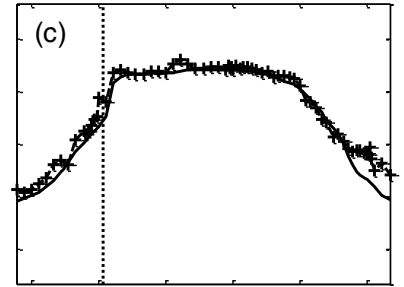

$\mathrm{A} 4 ; \mathrm{SE}=78.7 ; \mathrm{VA}=90 ; z=7 \mathrm{~m}$

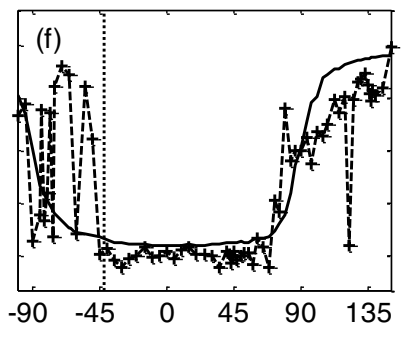

Fig. 14. AoLP outside the principal plane from CCNY polarimeter measurements (dashed curve with symbols) and from simulations (solid curves), with various solar elevation angles, viewing azimuth angles, and instrument depths. The AoLP is defined from $-90^{\circ}$ to $90^{\circ}$, as shown in Fig. 12(a).

in clear and close-to-surface waters, the light field is dominated by in-water single scattering. As a result, the polarization signals are largely determined by the single-scattering phase matrix. For example, the DoLP will be directly related to the $-S_{12} / S_{11}$ element if shown as a function of the scattering angle (the angle between the in-water solar and viewing directions for the first scattering event). As we showed in Subsection 5.A, the DoLP always peaks at a scattering angle of $94^{\circ}$, which is where the $-S_{12} / S_{11}$ element peaks, as well.

To understand why this is the case, we start from a derivation of the in-water single scattering. Generally, the scattered Stokes vector $\mathbf{I}$ is related to the incident Stokes vector $\mathbf{I}_{0}$ by

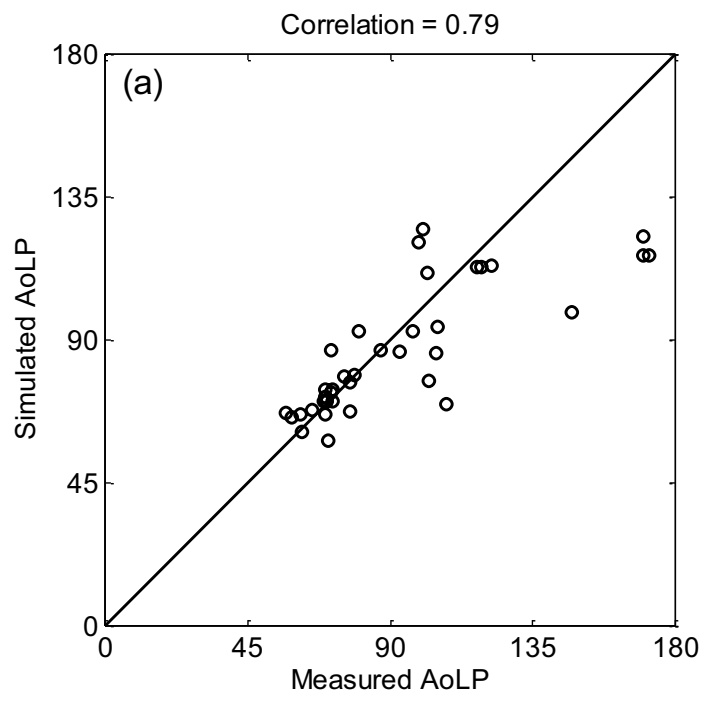

$$
\mathbf{I}(\theta, \phi)=\mathbf{L}\left(\sigma_{2}\right) \cdot \mathbf{P}(\Theta) \cdot \mathbf{L}\left(\sigma_{1}\right) \cdot \mathbf{I}_{0},
$$

where $\mathbf{P}(\Theta)$ is the single-scattering phase matrix with $\Theta$ being the scattering angle. $\mathbf{L}(\sigma)$ are rotation matrices that are required to change the plane of reference when the viewing direction is not in the principal plane, and are in the form of

$$
\mathbf{L}(\sigma)=\left[\begin{array}{cccc}
1 & 0 & 0 & 0 \\
0 & \cos (2 \sigma) & \sin (2 \sigma) & 0 \\
0 & -\sin (2 \sigma) & \cos (2 \sigma) & 0 \\
0 & 0 & 0 & 1
\end{array}\right]
$$

The two rotation angles are related to the incident and scattered directions by
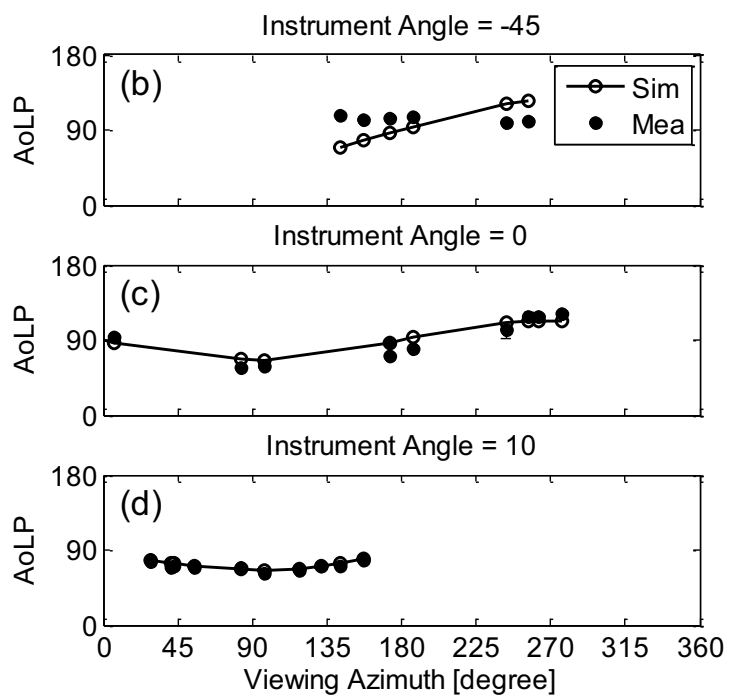

Fig. 15. Same as Fig. $\underline{9}$, but shows the AoLP [defined from $0^{\circ}$ to $180^{\circ}$, as shown in Fig. $\underline{12(b)}$ ]. 


$$
\begin{aligned}
& \cos \sigma_{1}=\frac{\cos \theta_{2}-\cos \theta_{1} \cos \Theta}{\sin \theta_{1} \sin \Theta}, \\
& \cos \sigma_{2}=\frac{\cos \theta_{1}-\cos \theta_{2} \cos \Theta}{\sin \theta_{2} \sin \Theta},
\end{aligned}
$$

where $\theta_{1}$ and $\theta_{2}$ are the incident and scattering zenith angles, respectively. For an unpolarized incident beam, $\mathbf{I}_{0}=[1,0,0,0]$, the above equations leads to

$$
\mathbf{I}(\theta, \phi)=\left[S_{11}(\Theta), S_{12}(\Theta) \cos \left(2 \sigma_{2}\right), S_{12}(\Theta) \sin \left(2 \sigma_{2}\right), 0\right]^{T}
$$

One can recognize now that the DoLP is identical to the $-S_{12} / S_{11}$ element, considering that $S_{12}$ is always negative. On the other hand, the AoLP is shown to be equal to the rotation angle $\sigma_{2}$, which solely depends on the solar and viewing geometry, and does not depend on the single-scattering Mueller matrix at all.

We note that efforts have been put toward exploring the possibility of predicting underwater polarization patterns using single-scattering calculations (see, for example, [2,9,12]). The previous singlescattering model for Do $\overline{\mathrm{LP}}$ was based on Rayleigh scattering, which corresponds to our case with a vanishing depolarization ratio $\rho$ [Eq. (3)] and a $-S_{12} / S_{11}$ pattern that peaks at $90^{\circ}$. Previous single-scattering models for AoLP \{see, for example, Eq. (1) in [9]\} can be shown to be identical to our formula (i.e., $\chi=\sigma_{2}$ ), but ours shows a clearer physical picture.

However, the incident light for the first in-water scattering event is not unpolarized. It is already linearly polarized due to Fresnel refraction, whose effect can be described by the following phase matrix (see, for example, [15]):

$$
\mathbf{F}=\left[\begin{array}{cccc}
\frac{1}{2}\left(a^{2}+b^{2}\right) & \frac{1}{2}\left(a^{2}-b^{2}\right) & 0 & 0 \\
\frac{1}{2}\left(a^{2}-b^{2}\right) & \frac{1}{2}\left(a^{2}+b^{2}\right) & 0 & 0 \\
0 & 0 & a b & 0 \\
0 & 0 & 0 & a b
\end{array}\right]
$$

where

$$
a=\frac{2 \cos \theta_{i}}{n \cos \theta_{i}+\cos \theta_{r}}, \quad b=\frac{2 \cos \theta_{i}}{\cos \theta_{i}+n \cos \theta_{r}},
$$

with $\theta_{i}$ and $\theta_{r}$ being the in-air and in-water solar zenith angles, and $n$ being the refractive index of water. Equation (4) now becomes

$$
\mathbf{I}(\theta, \phi)=\mathbf{L}\left(\sigma_{2}\right) \cdot \mathbf{P}(\Theta) \cdot \mathbf{L}\left(\sigma_{1}\right) \cdot \mathbf{F} \cdot \mathbf{I}_{0} .
$$

With this considered, the Stokes vector after the first in-water scattering event becomes more complicated and the scattered $Q$ and $U$ components become a function of angles $\Theta, \sigma_{1}$, and $\sigma_{2}$. It turns out that the DoLP and AoLP due to in-water single scattering depend not only on the scattering angle but also on the solar and viewing angles.

To demonstrate how well the single-scattering approximations work, we show measurements of DoLP (Fig. 16) and AoLP (Fig. 17) at selected stations as a function of the scattering angle along with predictions given by the single-scattering model without Fresnel refraction [gray solid curves, Eq. (7)] and the single-scattering model with Fresnel refraction [blue dashed curves, Eq. (10)]. The DoLP predictions

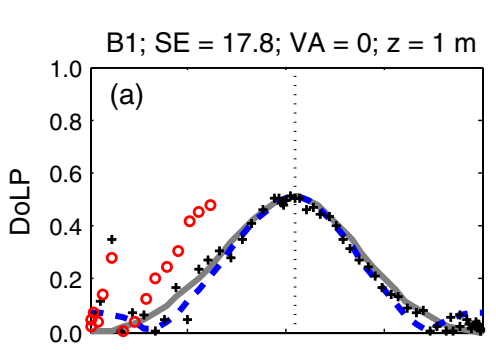

$510 \mathrm{~nm}$

$B 2 ; \mathrm{SE}=21.0 ; \mathrm{VA}=45 ; \mathrm{z}=1 \mathrm{~m}$

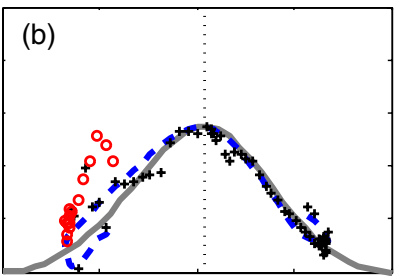

$B 8 ; S E=57.7 ; V A=270 ; z=7 m$
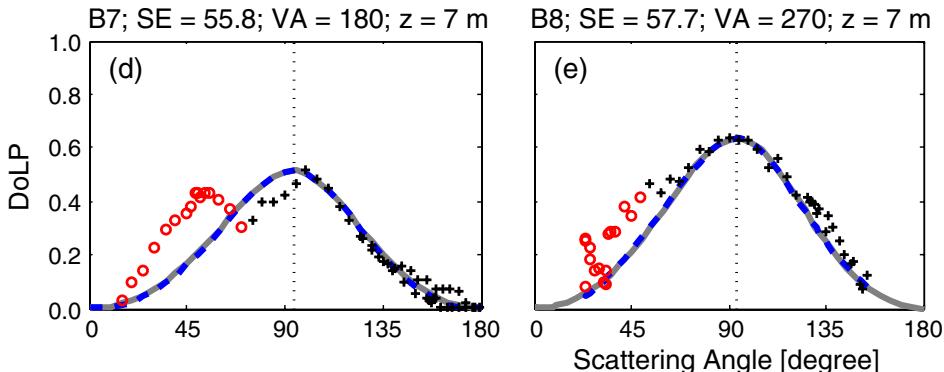

$B 3 ; \mathrm{SE}=25.1 ; \mathrm{VA}=270 ; \mathrm{z}=1 \mathrm{~m}$

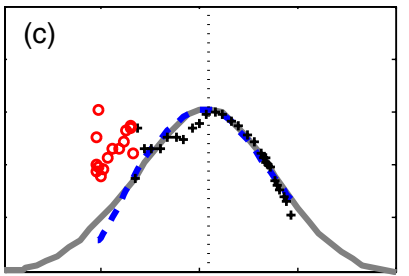

A3; $S E=74.4 ; V A=0 ; z=7 m$

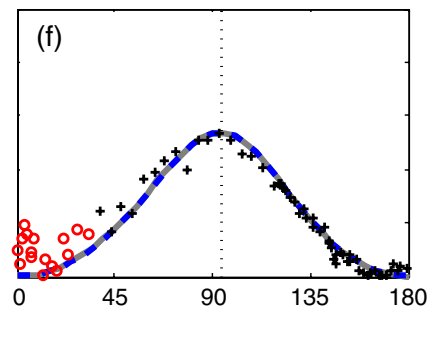

Fig. 16. (Color online) Measured DoLP (symbols) at selected stations as a function of the scattering angle. Black crosses indicate viewing angles out of Snell's cone; red circles indicate viewing angles in Snell's cone. Predictions by single-scattering approximations are also shown for comparison. Gray solid curves, without Fresnel refraction; blue dashed curves, with Fresnel refraction. Both are rescaled to match the maximum value in the measurements. 
$510 \mathrm{~nm}$

$B 2 ; \mathrm{SE}=21.0 ; \mathrm{VA}=45 ; \mathrm{z}=1 \mathrm{~m}$

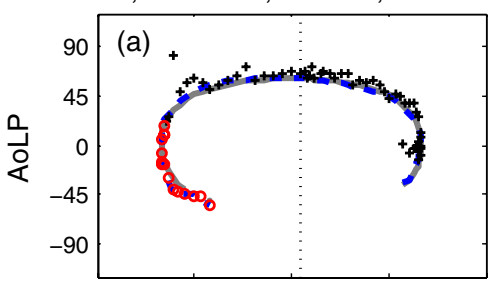

$B 6 ; S E=42.0 ; V A=270 ; z=1 \mathrm{~m}$

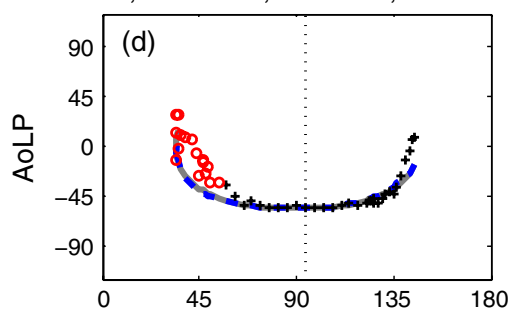

$B 3 ; \mathrm{SE}=25.1 ; \mathrm{VA}=270 ; \mathrm{z}=1 \mathrm{~m}$

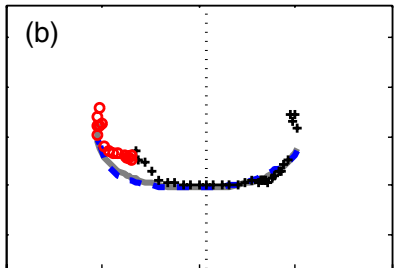

$B 8 ; S E=57.7 ; V A=270 ; z=7 m$

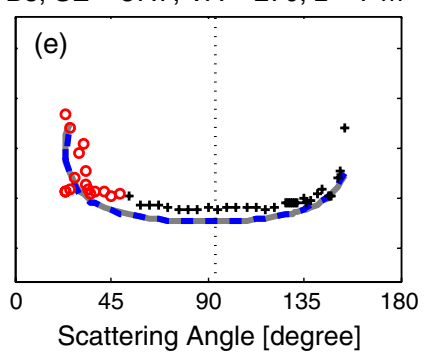

$\mathrm{B} 5 ; \mathrm{SE}=32.2 ; \mathrm{VA}=45 ; \mathrm{z}=1 \mathrm{~m}$

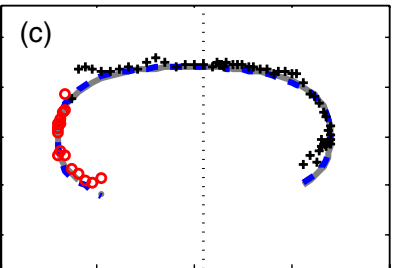

$\mathrm{A} 4 ; \mathrm{SE}=78.7 ; \mathrm{VA}=270 ; \mathrm{z}=7 \mathrm{~m}$

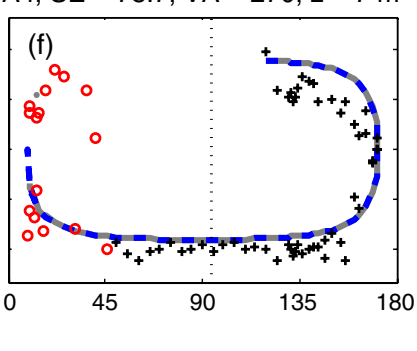

Fig. 17. (Color online) Same as Fig. 16, but shows measured AoLP at selected non-principal-plane stations as compared with single-scattering approximations.

are rescaled such that the maximum value equals to that in the measured DoLP. Although the DoLP is significantly lower than the $-S_{12} / S_{11}$ element due to depolarization of the single-scattering signal by higher-order scatterings, the single-scattering approximation without Fresnel refraction (gray solid curves) does give surprisingly good predictions of the overall shape of the DoLP pattern, as long as the viewing direction is outside Snell's cone (black crosses). Inside Snell's cone (red circles), the single-scattering approximation gives poor predictions since, in that case, the transmitted skylight dominates (see, for example, [8,41]). The single-scattering model with Fresnel refraction (blue dashed curves) gives as good predictions when the solar elevation is higher than $50^{\circ}$. However, for lower solar elevations, it gives poor predictions. This implies that in-water multiple scattering comes into play and ren-

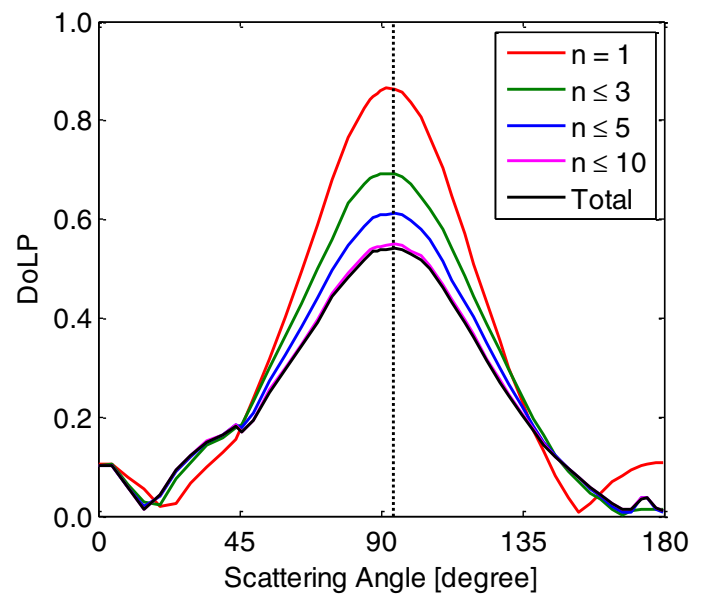

Fig. 18. (Color online) Effects of multiple scattering on the inwater DoLP pattern as a function of the scattering angle. The order of scattering is denoted by $n$. Geometries and optical properties for station B1 are used. ders the total polarization signal more similar to the case predicted by the single-scattering model without Fresnel refraction, which has been used in the community for decades.

On the other hand, the two single-scattering models give the same predictions to AoLP patterns (Fig. 17), which are generally in good agreement with measurements both inside and outside Snell's cone. This implies that the underwater e-vector orientation is primarily determined by single-scattering events, and the existence of the Fresnel interface does not have a significant effect on it.

To show the effects of multiple scattering, in Fig. 18, we show DoLP patterns from a Monte Carlo computation where contributions from various orders of scattering events are shown separately. For example, the red curve shows the DoLP pattern when only the first order of scattering event is included $(n=1)$, the blue curve when only the first three orders of scattering event are included $(n \leqslant 3)$, etc. Here $n$ is the order of scattering in the atmosphere or the ocean. The black curve shows the DoLP pattern for the total light field for comparison. The first-order result is similar to that from the singlescattering model with Fresnel refraction. As higher-order contributions are included, the maximum value of DoLP decreases, and the overall DoLP pattern moves toward that predicted by the singlescattering model without Fresnel refraction. The DoLP pattern given by the first 10 orders of scattering is almost identical to the total DoLP pattern.

The above measurement results, however, do not show obvious sensitivity of the maximum value of DoLP on various environmental parameters, such as the solar elevation, water turbidity, or detector depth, since the measurement data do not cover a sufficient range of these parameters. It is more convenient to do sensitivity studies on simulation 
results. To better analyze the dependence of maximum DoLP on these parameters, we made simulation runs with various solar elevations, detector depths, and water turbidities. Figure 19 shows results from these model simulations for various solar
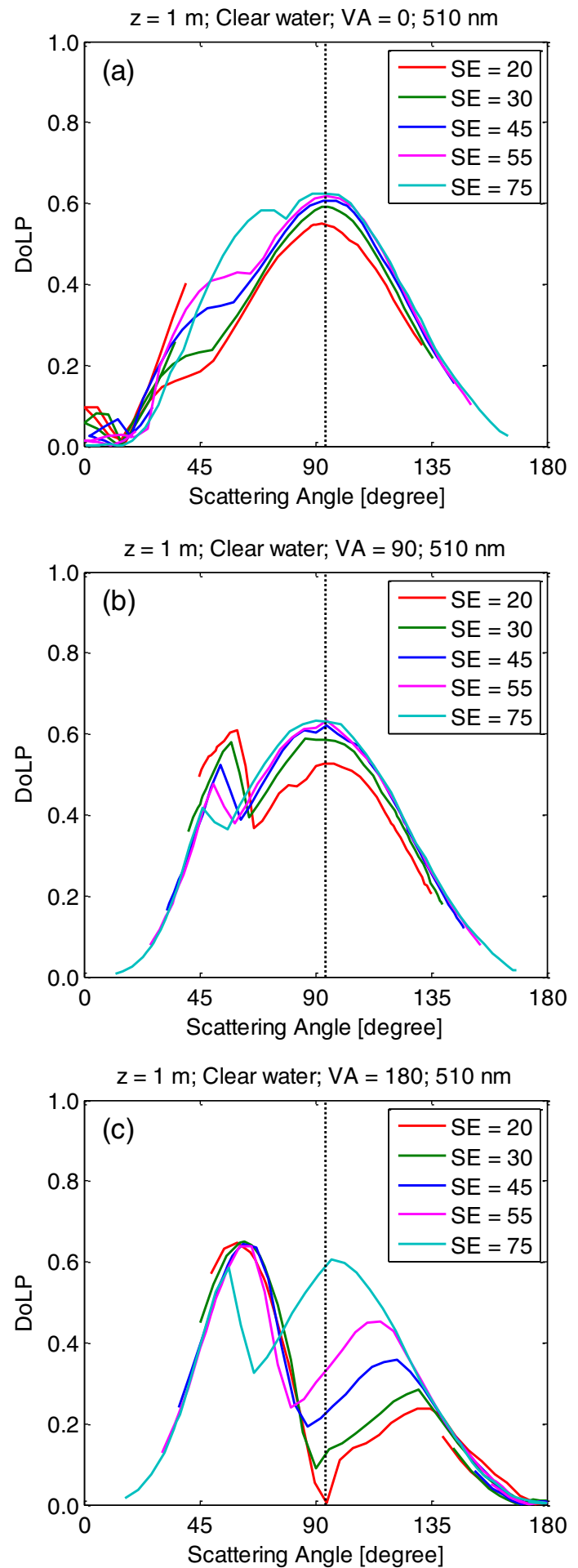

Fig. 19. (Color online) DoLP as a function of the scattering angle for various solar elevations at $1 \mathrm{~m}$ below surface in the clear water case (water IOPs and VSF from station B1). (a) Viewing azimuth $=0^{\circ}$, (b) viewing azimuth $=90^{\circ}$, and (c) viewing azimuth $=180^{\circ}$. elevations at $1 \mathrm{~m}$ below surface in the clear water case (water IOPs and VSF from station B1). At viewing azimuth angles of $0^{\circ}$ and $90^{\circ}$, a main DoLP peak at the scattering angle of $94^{\circ}$ is observed. In the solar half of the principal plane $\left(\mathrm{VA}=0^{\circ}\right)$, there is a secondary peak at around scattering angle $45^{\circ}$ [which is out of Snell's cone, see Figs. 7(a) and 7(d)] that is building up as the solar elevation increases. In the perpendicular plane $\left(\mathrm{VA}=90^{\circ}\right)$, there is an abrupt discontinuity around the same scattering angle [which is across the edge of Snell's cone, see Figs. 7(c) and 7(f)]. Inside the Snell's cone, the DoLP is as high as 0.6 , which decreases as the solar elevation increases. Interestingly, in the antisolar half of the principal plane $\left(\mathrm{VA}=180^{\circ}\right)$, the main peak appears at a scattering angle much larger than $94^{\circ}$ for a low solar elevation, and moves back toward $94^{\circ}$ as the solar elevation increases [note that this feature was not obvious in the measurement data shown in Fig. 16(d) since the solar elevation there was $55.8^{\circ}$ and the peak appeared very close to $94^{\circ}$ ]. On the other hand, a high DoLP value of 0.6 is always present just inside Snell's cone [see Fig. 9(b)]. For all three viewing azimuth angles, there is a higher DoLP at the main peak for a higher solar elevation. This general dependence on the solar elevation agrees with that reported by [2], and was not as obvious by looking at the measurement data (Fig. 7). Results shown here also suggest that the simple single-scattering model, which failed to predict the dependence of the DoLP on solar elevation and viewing azimuth angles, has its limitations.

Figure 20 shows simulated DoLP and AoLP patterns for the lowest and highest solar elevations, viewing azimuth angle of $0^{\circ}$, but with various detector depths ( $1 \mathrm{~m}$ in solid curves and $7 \mathrm{~m}$ in dotted curves) and water turbidities [clear water in Figs. 20(a) and 20(c) and turbid water in panels Figs. $20(\mathrm{~b})$ and $2 \overline{0(\mathrm{~d})]}$. These simulation results suggest that the maximum DoLP decreases obviously when the water turbidity increases. On the other hand, the dependence on the detector depth is marginal, with the maximum DoLP at $7 \mathrm{~m}$ slightly lower than that at $1 \mathrm{~m}$. Both dependences can be attributed to multiple scattering in the water that depolarizes the light. Another significant feature is that the main DoLP peak appears at a scattering angle smaller than $94^{\circ}$ for the lowest solar elevation, $20^{\circ}$. This phenomenon did not show up in the measurement data, as the Sun was always higher than $40^{\circ}$ at turbid water stations (Table 1). Simulation results show that, for the water IOP $\overline{\mathrm{P}}_{\mathrm{s}}$ measured at A stations, the DoLP peak starts moving to smaller scattering angles when the Sun is lower than $30^{\circ}$ (results not shown). This is because effects from multiply scattered light start to show up when the water turbidity (more precisely, water scattering) or the path length along the incident direction becomes sufficiently large. Furthermore, the secondary peak [see Fig. 19(a)] disappears. The same dependences on depth and water turbidity apply to results for other 

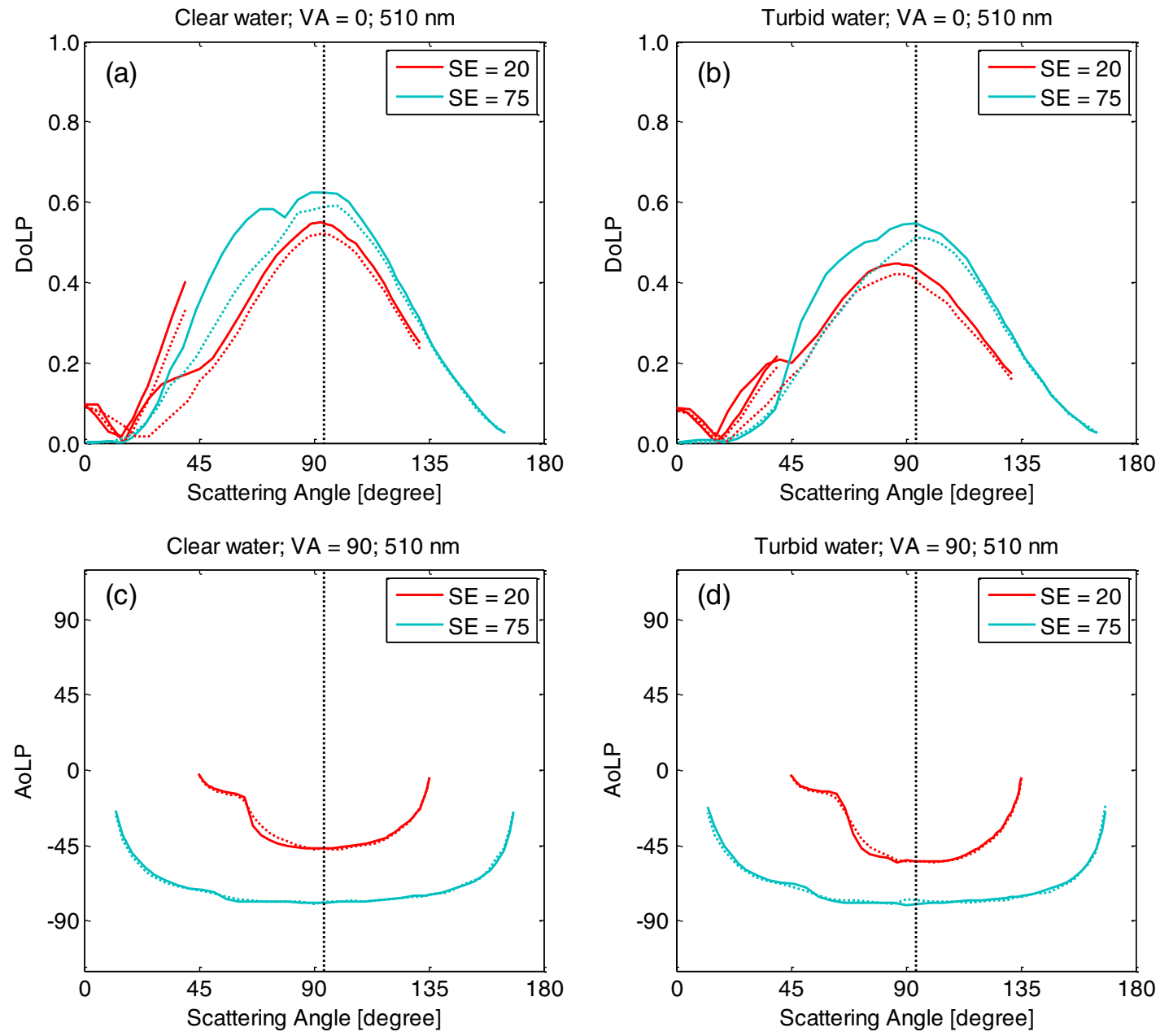

Fig. 20. (Color online) Same as Fig. 18, but shows DoLP and AoLP for various detector depths and water turbidities. (a) and (c) Clear water (water IOPs and VSF from station B1), and (b) and (d) turbid water (water IOPs and VSF from station A1). Solid curves, detector at $1 \mathrm{~m}$ below surface; dotted curves, $7 \mathrm{~m}$ below surface.

viewing azimuth angles (results not shown). The dependences of the DoLP on the water turbidity and detector depth are consistent with those previously reported [42].

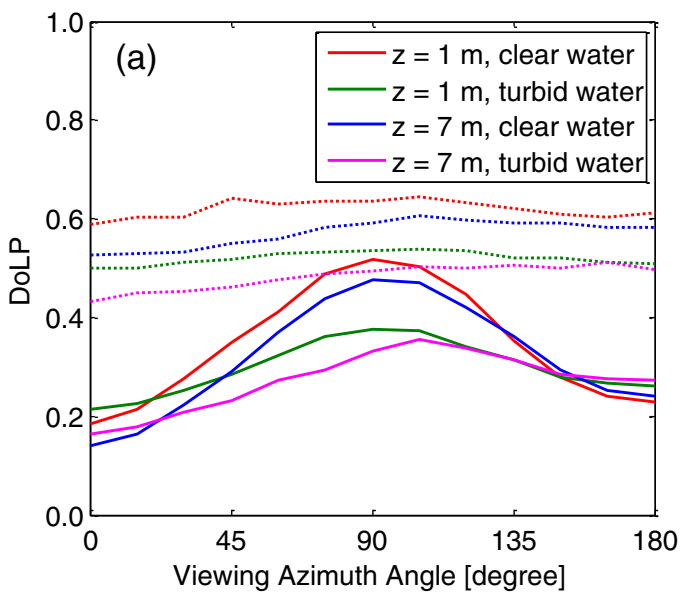

The simulated AoLP at a viewing azimuth angle of $90^{\circ}$ turns out to be highly sensitive to the solar elevation, with that for a higher elevation much further away from the meridian plane. However, it shows

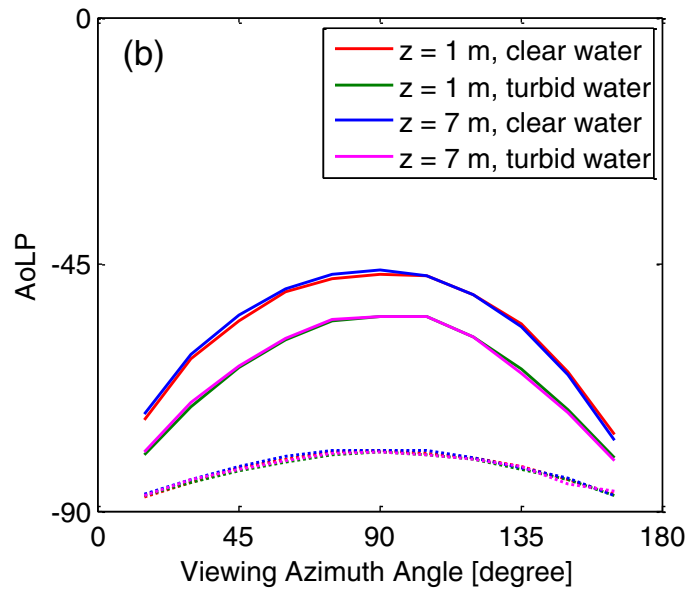

Fig. 21. (Color online) Simulated DoLP and AoLP along the horizontal viewing direction (an instrument angle of $0^{\circ}$ ) at various detector depths in the clear and turbid water conditions as a function of the viewing azimuth angle. Solid curves, solar elevation $=20^{\circ} ;$ dotted curves, solar elevation $=75^{\circ}$. 
little dependence on the water turbidity at the lowest solar elevation, $20^{\circ}$, with that in the turbid water being roughly $6^{\circ}$ farther away from the meridian plane. For the highest solar elevation, $75^{\circ}$, the AoLP shows almost no dependence on the water turbidity. Also, the AoLP patterns at the two depths are almost identical. These observations apply to simulated AoLP patterns for viewing azimuth angles $45^{\circ}$ and $135^{\circ}$ as well, with the only exception that, when $\mathrm{VA}=45^{\circ}$ and $\mathrm{SE}=20^{\circ}$, the AoLP in the clear water is farther away from the meridian plane (results not shown).

In addition to the above dependence studies, it is of specific interest to look at the polarization signals in horizontal viewing directions, since most animals are horizontal viewing. In Fig. 21, we show the simulated DoLP and AoLP along horizontal viewing directions (an instrument angle of $0^{\circ}$ ) as a function of the viewing azimuth angle. As suggested by results in Fig. 21(a), for a low solar elevation (solid curves), the DoLP is highly dependent on the viewing azimuth angle. This implies that animals can use the DoLP signal for orientation. On the other hand, for a high solar elevation (dotted curves), the DoLP becomes almost independent to the viewing azimuth angle. The previously observed dependence on the detector depth and water turbidity can also be seen here. This dependence on the viewing azimuth angle at low and high solar elevations applies to the AoLP, as well [Fig. 21(b)].

\section{Conclusions}

In this paper, we report measurements of the underwater polarized light field acquired during a field experiment in open ocean environments near Port Aransas, Texas, using two custom-built instruments: a hyperspectral and multiangular polarimeter developed by the Optical Remote Sensing Laboratory at the City College of New York, and a prototype video polarimeter developed by the University of Texas at Austin. The measured polarization characteristics of the underwater light field, including the normalized $Q$ and $U$ components and the degree and angle of linear polarization, were then compared against their counterparts from radiative transfer model simulations using a vector Monte Carlo method. Concurrent measurements of water IOPs including particulate VSFs were used as input to model simulations. Comparisons between spectral polarization states from simulations and from the CCNY polarimeter measurements show good agreement for a variety of sky conditions, water conditions, surface conditions, solar elevations, viewing directions, and instrument depths. Discrepancies were observed in red wavelengths, where water Raman scatter and chlorophyll fluorescence due to phytoplankton particles were not correctly represented in the radiative transfer model. Despite low concentrations of chlorophyll $a$ $\left(0.2 \mathrm{mg} / \mathrm{m}^{3}\right)$ the relative dip of DoLP due to chlorophyll fluorescence is quite pronounced and was found to be of the order of $25 \%-30 \%$. Degree and angle of linear polarization measured by the UT video polarimeter were also compared with modeling results. The measured and simulated angles of linear polarization were highly consistent, while the measured DoLP was correlated to, but in general lower than, the simulations. These comparisons verify that measured linear polarization states, including the DoLP and AoLP, from the two instruments are generally reliable in most cases. Our results also revealed that an agreement between measured and simulated DoLP does not necessarily correspond to an agreement between measured and simulated full linear polarization states. Both DoLP and AoLP must be considered together.

Our measurements of the DoLP in the ocean were comparable to those reported elsewhere. Our maxima of $65 \%$ [Figs. 7(f) and 9(c)] is higher than those measured in coastal New York waters (Tonizzo [14]), in the range of those reported for coral reef environments (Cronin and Shashar [7], Sabbah et al. [43]), and lower than the early data reported by Ivanoff et al. [4]. Similar to the work by Cronin and Shashar [7], our measurements of the angle of polarized light demonstrate the complexity of the e-vector orientation as it differs with solar elevation and viewing angle. As Waterman [44] eloquently argued, the e-vector orientation is rarely dominated by a horizontal plane of polarization when the Sun is not directly overhead, and this was observed at both 1 and $7 \mathrm{~m}$ depths (Fig. 15). Such a complex e-vector environment can lead to challenges for animals needing to camouflage or hide from predators with polarization vision, as the polarization field varies dramatically with the spherical viewing angles $(\theta$ and $\varphi)$. Conversely, this environment provides rich opportunity for animals to develop communication signals that can stand out against the background polarization field. Furthermore, polarization-sensitive marine organisms may take advantage of these polarization patterns to locate the Sun's azimuth angle, which could be used as an underwater compass for navigation (Waterman [44]). Our measurements of the polarization light field provide the first step in identifying the environmental parameters that polarization-sensitive organisms face as constraints in evolving effective polarization communication and camouflage traits.

The measured underwater DoLP and AoLP as a function of the scattering angle are analyzed. In some cases, they can both be predicted by a simple in-water single-scattering model without considering the Fresnel refraction at the air-sea surface, which has been used in the literature for decades. However, our analyses indicate that this model works because the effects of Fresnel refraction and multiple scattering cancel with each other. Specifically, in measurements outside of Snell's cone, the single-scattering and the fully multiple scattering models, the DoLP always peaks at a scattering angle of $94^{\circ}$ (Fig. 16).

The dependence of underwater DoLP and AoLP on various parameters have been studied based on model-simulated polarized light fields. The 
maximum value of DoLP is found to increase when the solar elevation increases, the water turbidity decreases, and the detector depth decreases (Figs. 19 and 20). These findings are consistent with those reported in previous studies. The simulated DoLP pattern at horizontal viewing directions is highly dependent on the viewing azimuth angle for a low solar elevation. This implies that animals can use the DoLP signal for orientation.

This work was supported by a Department of Defense (DOD) Office of Naval Research (ONR) Multi-University Research Initiative (MURI) grant N000140911054 and also an ONR grant N0001406-1-0069. The CCNY group also acknowledges a partial support from National Oceanic and Atmospheric Administration (NOAA) grant NA06OAR 4810187 and another ONR grant N000141010368. We acknowledge Amir Ibrahim, Tara Maginnis, Rick Kline, Frank Ernst, Kaylan Randolph, Heather Groundwater, Brandon Russell, Richard Zimmerman, and Scott Freeman for their field work. We also thank Lee Fuiman, Steve Lanoux, and especially Frank Ernst, for logistical support from UT's Marine Science Institute at Port Aransas. We also acknowledge NASA Ocean Color Processing Group for imagery. Y. You acknowledges Shouguo Ding for making LBLRTM calculations. We also thank the editor and anonymous reviewers for their valuable comments and suggestions.

\section{References}

1. T. H. Waterman, "Polarization patterns in submarine illumination," Science 120, 927-932 (1954).

2. T. H. Waterman and W. E. Westell, "Quantitative effects of the sun's position on submarine light polarization," J. Mar. Res. 15, 149-169 (1956).

3. A. Ivanoff and T. H. Waterman, "Elliptical polarization of submarine illumination," J. Mar. Res. 16, 283-307 (1958).

4. A. Ivanoff, N. Jerlov, and T. H. Waterman, "A comparative study of irradiance, beam transmittance and scattering in the sea near Bermuda," Limnol. Oceanogr. 6, 129-148 (1961).

5. V. A. Timofeeva, "Optics of turbid waters (results of laboratory studies)," in Optical Aspects of Oceanography, N. Jerlov and E. Nielsen, eds. (Academic, 1974), pp. 177-219.

6. M. Chami, "Importance of the polarization in the retrieval of oceanic constituents from the remote sensing reflectance," J. Geophys. Res. Oceans 112, C05026 (2007).

7. T. W. Cronin and N. Shashar, "The linearly polarized light field in clear, tropical marine waters: spatial and temporal variation of light intensity, degree of polarization and e-vector angle," J. Exp. Biol. 204, 2461-2467 (2001).

8. S. Sabbah, A. Barta, J. Gál, G. Horváth, and N. Shashar, "Experimental and theoretical study of skylight polarization transmitted through Snell's window of a flat water surface," J. Opt. Soc. Am. A 23, 1978-1988 (2006).

9. S. Sabbah and N. Shashar, "Light polarization under water near sunrise," J. Opt. Soc. Am. A 24, 2049-2056 (2007).

10. N. Shashar, S. Sabbah, and T. W. Cronin, "Transmission of linearly polarized light in seawater: implications for polarization signaling," J. Exp. Biol. 207, 3619-3628 (2004).

11. S. Johnsen, N. J. Marshall, and E. A. Widder, "Polarization sensitivity as a contrast enhancer in pelagic predators: lessons from in situ polarization imaging of transparent zooplankton," Phil. Trans. R. Soc. B 366, 655-670 (2011).
12. A. Lerner, S. Sabbah, C. Erlick, and N. Shashar, "Navigation by light polarization in clear and turbid waters," Phil. Trans. R. Soc. B 366, 671-679 (2011).

13. N. Shashar, S. Johnsen, A. Lerner, S. Sabbah, C. C. Chiao, L. M. Mathger, and R. T. Hanlon, "Underwater linear polarization: physical limitations to biological functions," Phil. Trans. R. Soc. B 366, 649-654 (2011).

14. A. Tonizzo, J. Zhou, A. Gilerson, M. S. Twardowski, D. J. Gray, R. A. Arnone, B. M. Gross, F. Moshary, and S. A. Ahmed, "Polarized light in coastal waters: hyperspectral and multiangular analysis," Opt. Express 17, 5666-5682 (2009).

15. G. W. Kattawar and C. N. Adams, "Stokes vector calculations of the submarine light-field in an atmosphere-ocean with scattering according to a Rayleigh phase matrix-effect of interface refractive-index on radiance and polarization," Limnol. Oceanogr. 34, 1453-1472 (1989).

16. G. W. Kattawar, G. N. Plass, and J. A. Guinn, Jr., "Monte Carlo calculations of the polarization of radiation in the Earth's atmosphere-ocean system," J. Phys. Oceanogr. 3, 353-372 (1973).

17. P.-W. Zhai, G. W. Kattawar, and P. Yang, "Impulse response solution to the three-dimensional vector radiative transfer equation in atmosphere-ocean systems. I. Monte Carlo method," Appl. Opt. 47, 1037-1047 (2008).

18. H. H. Tynes, G. W. Kattawar, E. P. Zege, I. L. Katsev, A. S. Prikhach, and L. I. Chaikovskaya, "Monte Carlo and multicomponent approximation methods for vector radiative transfer by use of effective Mueller matrix calculations," Appl. Opt. 40, 400-412 (2001).

19. J. T. Adams, E. Aas, N. K. Hojerslev, and B. Lundgren, "Comparison of radiance and polarization values observed in the Mediterranean Sea and simulated in a Monte Carlo model," Appl. Opt. 41, 2724-2733 (2002).

20. G. W. Kattawar and G. N. Plass, "Degree and direction of polarization of multiple scattered light. 1. Homogeneous cloud layers," Appl. Opt. 11, 2851-2865 (1972).

21. G. W. Kattawar, "Polarization of light in the sea," in Ocean Optics, R. W. Spinrad, K. L. Carder, and M. J. Perry, eds. (Oxford University, 1994), pp. 202-219.

22. M. S. Twardowski, J. M. Sullivan, P. L. Donaghay, and J. R. V. Zaneveld, "Microscale quantification of the absorption by dissolved and particulate material in coastal waters with an ac9," J. Atmos. Ocean. Technol. 16, 691-707 (1999).

23. D. McKee, J. Piskozub, and I. Brown, "Scattering error corrections for in situ absorption and attenuation measurements," Opt. Express 16, 19480-19492 (2008).

24. J. M. Sullivan and M. S. Twardowski, "Angular shape of the oceanic particulate volume scattering function in the backward direction," Appl. Opt. 48, 6811-6819 (2009).

25. K. N. Liou, An Introduction to Atmospheric Radiation, 2nd ed. (Academic, 2002).

26. J. Lenoble and C. Broquez, "A comparative review of radiation aerosol models," Contrib. Atmos. Phys. 57, 1-20 (1984).

27. S. A. Clough and M. J. Iacono, "Line-by-line calculations of atmospheric fluxes and cooling rates 2. Application to carbon dioxide, ozone, methane, nitrous oxide, and the halocarbons," J. Geophys. Res. 100, 16519-16535 (1995).

28. C. Cox and W. Munk, "Statistics of the sea surface derived from sun glitter," J. Mar. Res. 13, 198-227 (1954).

29. X. Zhang, L. Hu, and M.-X. He, "Scattering by pure seawater: effect of salinity," Opt. Express 17, 5698-5710 (2009).

30. R. M. Pope and E. S. Fry, "Absorption spectrum $(380-700 \mathrm{~nm})$ of pure water. 2. Integrating cavity measurements," Appl. Opt. 36, 8710-8723 (1997).

31. H. C. van de Hulst, Light Scattering by Small Particles (Dover, 1981). 
32. S. Chandrasekhar, Radiative Transfer (Dover, 1960).

33. K. J. Voss and E. S. Fry, "Measurement of the Mueller matrix for ocean water," Appl. Opt. 23, 44274439 (1984).

34. J. E. Hansen and L. D. Travis, "Light scattering in planetary atmospheres," Space Sci. Rev. 16, 527-610 (1974).

35. G. C. Feldman and C. R. McClain, Ocean Color Web, MODerate-resolution Imaging Spectroradiometer (MODIS) Aqua Sensor Reprocessing 2009.1, N. Kuring and S. W. Bailey, eds. (NASA Goddard Space Flight Center, 4 Dec. 2010), http:// oceancolor.gsfc.nasa.gov/.

36. C. D. Mobley, "HydroLight Users' Guide," http://www .sequoiasci.com/downloads/HE5UsersGuide.pdf.

37. T. J. Petzold, Volume Scattering Functions for Selected Ocean Waters (Scripps Institution of Oceanography, 1972).

38. L. G. Henyey and J. L. Greenstein, "Diffuse radiation in the galaxy," Astrophys. J. 93, 70-83 (1941).

39. H. R. Gordon, M. R. Lewis, S. D. McLean, M. S. Twardowski, S. A. Freeman, K. J. Voss, and G. C. Boynton, "Spectra of particulate backscattering in natural waters," Opt. Express 17, 16192-16208 (2009).

40. A. Tonizzo, J. Zhou, A. Gilerson, B. Gross, F. Moshary and S. Ahmed, "The impact of algal fluorescence on the underwater polarized light field," Proc. SPIE 7678, 76780K (2010).

41. G. Horvath and D. Varju, "Underwater refraction-polarization patterns of skylight perceived by aquatic animals through Snell's window of the flat water-surface," Vision Res. 35, 1651-1666 (1995).

42. A. Ivanoff, "Polarization measurements in the sea," in Optical Aspects of Oceanography, N. Jerlov and E. Nielsen, eds. (Academic, 1974), pp. 151-176.

43. S. Sabbah, A. Lerner, C. Erlick, and N. Shashar, "Under water polarization vision-a physical examination," in Recent Research Developments in Experimental \& Theoretical Biology, S. G. Pandalai, ed. (Transworld Research Network, 2005), Vol. 1, pp. 123-176.

44. T. H. Waterman, "Reviving a neglected celestial underwater polarization compass for aquatic animals," Biol. Rev. 81, 111-115 (2006). 